ANEXO I - Média dos erros absoluto, variável e constante, dos participantes dos grupos do experimento 1 (tarefa simples), dos grupos CO, AL, CO-LA e AL-CO, dos oito blocos de tentativas da fase de estabilização (E1 a E8) e dos quatro blocos de tentativas da fase de adaptação (A1, A2, A3 e A4), em milissegundos.

\title{
Erro absoluto
}

\begin{tabular}{|c|c|c|c|c|c|c|c|c|c|c|c|c|}
\hline \multicolumn{13}{|l|}{$\mathrm{CO}$} \\
\hline 1 & 214,00 & 71,50 & 33,38 & 154,25 & 527,13 & 427,75 & 542,25 & 565,78 & 519,38 & 580,11 & 839,43 & 327,14 \\
\hline 2 & 67,60 & 113,00 & 26,00 & 124,00 & 55,22 & 77,00 & 79,43 & 73,25 & 84,50 & 103,50 & 91,57 & 61,83 \\
\hline 3 & 1133,63 & 139,71 & 156,43 & 242,33 & 139,43 & 147,75 & 96,00 & 160,00 & 194,50 & 171,56 & 183,75 & 320,14 \\
\hline 4 & 134,00 & 69,13 & 190,40 & 107,00 & 94,80 & 53,00 & 53,40 & 103,67 & 72,67 & 297,75 & 191,75 & 136,83 \\
\hline 5 & 244,33 & 79,00 & 57,50 & 67,71 & 87,67 & 105,00 & 69,88 & 109,86 & 175,50 & 137,00 & 94,00 & 235,00 \\
\hline 6 & 122,75 & 213,00 & 55,57 & 95,17 & 77,20 & 187,29 & 38,67 & 93,60 & 213,40 & 157,86 & 99,00 & 96,83 \\
\hline 7 & 69,00 & 117,25 & 62,13 & 67,13 & 65,75 & 60,25 & 100,00 & 97,11 & 66,67 & 75,43 & 71,11 & 161,88 \\
\hline 8 & 877,33 & 191,33 & 78,00 & 24,00 & 38,20 & 117,86 & 229,00 & 149,29 & 85,17 & 107,33 & 120,50 & 128,17 \\
\hline 9 & 282,88 & 252,56 & 205,78 & 198,63 & 56,56 & 59,75 & 54,75 & 168,00 & 118,00 & 163,00 & 110,13 & 192,80 \\
\hline 10 & 118,40 & 82,33 & 343,17 & 219,60 & 195,00 & 186,00 & 204,13 & 365,83 & 606,43 & 192,14 & 62,00 & 193,00 \\
\hline 11 & 115,22 & 104,00 & 78,50 & 70,43 & 69,13 & 143,56 & 70,67 & 67,56 & 73,78 & 107,44 & 96,22 & 232,89 \\
\hline 12 & 166,80 & 114,57 & 157,00 & 97,67 & 164,89 & 145,00 & 99,63 & 102,50 & 109,43 & 137,25 & 77,44 & 76,22 \\
\hline 13 & 192,88 & 104,75 & 68,13 & 53,14 & 69,78 & 83,67 & 68,75 & 107,67 & 70,38 & 89,00 & 105,50 & 140,44 \\
\hline 14 & 100,38 & 90,50 & 73,43 & 100,25 & 48,11 & 73,00 & 162,80 & 133,43 & 141,22 & 130,25 & 109,00 & 140,86 \\
\hline 15 & 281,14 & 112,44 & 100,44 & 54,75 & 97,00 & 80,78 & 44,29 & 73,11 & 198,56 & 159,56 & 268,50 & 324,00 \\
\hline 16 & 94,25 & 56,75 & 99,33 & 48,75 & 110,67 & 259,78 & 113,33 & 281,78 & 66,25 & 133,83 & 135,00 & 384,14 \\
\hline 17 & 145,80 & 240,57 & 124,11 & 98,00 & 120,20 & 108,75 & 72,38 & 202,11 & 52,86 & 258,00 & 247,00 & 286,71 \\
\hline 18 & 230,00 & 43,50 & 101,89 & 57,00 & 126,75 & 46,89 & 70,25 & 129,56 & 91,80 & 103,67 & 78,29 & 60,67 \\
\hline 19 & 64,50 & 90,63 & 50,75 & 70,11 & 53,56 & 85,11 & 82,38 & 69,33 & 75,33 & 137,63 & 52,00 & 78,71 \\
\hline 20 & 163,60 & 54,14 & 181,40 & 125,25 & 72,67 & 63,33 & 74,22 & 52,13 & 52,25 & 102,25 & 117,88 & 83,44 \\
\hline \multicolumn{13}{|l|}{$\mathrm{AL}$} \\
\hline 1 & 174,50 & 280,57 & 137,63 & 297,25 & 123,22 & 55,00 & 108,13 & 134,86 & 147,78 & 44,33 & 293,33 & 108,67 \\
\hline 2 & 113,00 & 83,83 & 95,63 & 101,44 & 98,44 & 102,00 & 136,50 & 40,57 & 45,33 & 101,86 & 68,56 & 69,63 \\
\hline 3 & 446,00 & 569,33 & 612,33 & 805,40 & 699,60 & 685,67 & 711,17 & 780,50 & 414,83 & 998,40 & 952,83 & 821,29 \\
\hline 4 & 219,63 & 150,29 & 124,33 & 85,50 & 116,50 & 50,17 & 48,67 & 62,38 & 59,63 & 133,13 & 118,11 & 50,63 \\
\hline 5 & 330,00 & 245,50 & 204,44 & 76,80 & 50,57 & 67,71 & 88,83 & 52,33 & 53,60 & 38,14 & 42,83 & 44,71 \\
\hline 6 & 33,71 & 58,88 & 101,86 & 64,50 & 97,25 & 40,75 & 77,13 & 69,71 & 63,00 & 104,67 & 39,29 & 59,88 \\
\hline 7 & 145,57 & 230,56 & 200,29 & 172,80 & 1057,33 & 505,56 & 297,89 & 131,38 & 149,67 & 191,67 & 69,00 & 124,44 \\
\hline 8 & 95,80 & 126,33 & 235,80 & 285,40 & 386,00 & 102,20 & 281,75 & 228,00 & 90,83 & 64,00 & 104,14 & 63,50 \\
\hline 9 & 65,00 & 65,17 & 71,25 & 61,22 & 77,88 & 72,00 & 85,67 & 87,17 & 79,78 & 106,22 & 105,22 & 112,89 \\
\hline 10 & 974,50 & 314,50 & 520,13 & 421,17 & 301,88 & 549,57 & 376,13 & 135,00 & 148,00 & 118,67 & 180,71 & 146,83 \\
\hline 11 & 364,00 & 79,86 & 124,63 & 83,22 & 92,50 & 75,44 & 92,13 & 126,89 & 92,88 & 117,11 & 140,83 & 159,00 \\
\hline 12 & 1186,29 & 315,83 & 203,88 & 409,89 & 265,88 & 360,44 & 150,88 & 194,67 & 136,86 & 141,40 & 72,86 & 198,38 \\
\hline 13 & 290,78 & 665,67 & 299,56 & 166,44 & 248,67 & 137,00 & 70,71 & 387,00 & 310,17 & 139,78 & 140,71 & 112,44 \\
\hline 14 & 468,00 & 391,50 & 245,78 & 301,60 & 203,89 & 259,00 & 150,38 & 174,25 & 128,88 & 111,22 & 189,22 & 148,89 \\
\hline 15 & 912,63 & 200,44 & 174,67 & 104,33 & 141,38 & 106,43 & 69,67 & 54,25 & 159,89 & 63,78 & 99,25 & 160,33 \\
\hline 16 & 173,44 & 78,00 & 49,25 & 63,17 & 92,00 & 95,22 & 67,75 & 123,50 & 92,11 & 75,75 & 74,50 & 54,89 \\
\hline 17 & 797,50 & 1060,50 & 1050,17 & 534,63 & 442,88 & 373,50 & 464,80 & 383,00 & 891,00 & 243,00 & 238,43 & 297,50 \\
\hline 18 & 354,13 & 174,78 & 143,00 & 136,50 & 175,29 & 275,00 & 191,00 & 211,63 & 192,44 & 393,88 & 158,56 & 115,89 \\
\hline 19 & 567,86 & 548,44 & 237,89 & 153,00 & 321,44 & 111,78 & 158,57 & 171,00 & 320,38 & 151,25 & 79,11 & 227,38 \\
\hline 20 & 842,71 & 672,22 & 513,75 & 405,14 & 277,75 & 266,75 & 329,00 & 169,22 & 170,56 & 108,00 & 67,89 & 89,7 \\
\hline
\end{tabular}


ANEXO I - continuação.

\begin{tabular}{|c|c|c|c|c|c|c|c|c|c|c|c|c|}
\hline \multicolumn{13}{|l|}{$D-A$} \\
\hline 1 & 22,00 & 39 & 3,50 & 1 & 29 & 140,11 & 72,89 & 14 & 88 & 36 & 11 & 51,2 \\
\hline 2 & 56,33 & 19,00 & 91,78 & 50,00 & 90,71 & 104,89 & 129,71 & 136,50 & 146,50 & 86,50 & 4,38 & 48,13 \\
\hline 3 & 23,33 & 58,22 & 47,44 & 96,00 & 81,50 & 491,86 & 68,14 & 158,17 & 37,00 & 268,83 & 46,00 & 51,86 \\
\hline 4 & 24,33 & 334,43 & 01,88 & 79,89 & 47,38 & 72,83 & 90,33 & 123,33 & 89,83 & 76,86 & 184,63 & 51,43 \\
\hline 5 & 70,86 & 125,80 & 04,14 & 68,25 & 119,00 & 24,60 & 181,67 & 131,00 & 75,33 & 52,25 & 4,38 & 4,33 \\
\hline 6 & 218,11 & 111,14 & 129,25 & 181,50 & 216,33 & 263,00 & 230,43 & 249,71 & 269,14 & 263,00 & 165,38 & 98,00 \\
\hline 7 & 49,25 & 179,50 & 210,56 & 203,67 & 219,00 & 344,33 & 505,71 & 207,29 & 294,50 & 186,71 & 174,13 & 207,57 \\
\hline 8 & 84,33 & 59,40 & 43,75 & 119,29 & 134,22 & 139,00 & 136,44 & 132,13 & 188,22 & 202,63 & 162,63 & 150,89 \\
\hline 9 & 166,13 & 61,56 & 56,56 & 80,11 & 383,60 & 403,00 & 338,00 & 127,38 & 151,33 & 126,50 & 296,71 & 279,25 \\
\hline 10 & 55,00 & 296,00 & 281,75 & 48,00 & 186,00 & 304,00 & 243,22 & 249,17 & 342,00 & 253,89 & 117,71 & 325,50 \\
\hline 11 & 80,29 & 62,57 & 67,56 & 80,25 & 162,38 & 84,71 & 45,50 & 59,63 & 145,13 & 114,67 & 126,00 & 183,57 \\
\hline 12 & 15,13 & 41,63 & 74,50 & 93,00 & 157,50 & 191,44 & 78,13 & 96,25 & 58,63 & 86,63 & 85,00 & 91,33 \\
\hline 13 & 67,67 & 44,29 & 163,33 & 72,00 & 187,00 & 164,57 & 66,00 & 152,25 & 72,33 & 50,22 & 89 & 58,22 \\
\hline 14 & 55,00 & 68,33 & 59,25 & 46,63 & 103,83 & 171,89 & 74,57 & 69,00 & 105,75 & 69,63 & 4,33 & 57,78 \\
\hline 15 & 28,71 & 138,78 & 37,78 & 60,44 & 58,63 & 42,63 & 125,67 & 46,67 & 11 & 89 & 88 & 1,33 \\
\hline 16 & 27,67 & 615,88 & 60,63 & 62,13 & 542,71 & 429,11 & 113,22 & 68,50 & 136,29 & 102,89 & 274,88 & 64,78 \\
\hline 17 & 57,22 & 51,75 & 106,89 & 120,00 & 34,00 & 34,57 & 46,33 & 39,33 & 34,38 & 58,50 & 20,63 & 36,67 \\
\hline 18 & 1,80 & 104,29 & 151,25 & 136,33 & 258,00 & 157,88 & 196,00 & 186,20 & 210,67 & 127,78 & 145,75 & 109,33 \\
\hline 19 & 2,00 & 114,43 & 78,63 & 161,43 & 540,40 & 266,60 & 333,14 & 242,75 & 98,29 & 48,80 & 44,00 & 166,57 \\
\hline 2 & 5,14 & 56 & 14,11 & 0 & 71 & 75 & 78 & 1 & 236,44 & 39 & 33 & 9,2 \\
\hline \multicolumn{13}{|l|}{ L-C } \\
\hline 1 & 98,50 & 175,13 & 00,43 & 97,11 & 89,11 & 66,13 & 72,67 & 99,63 & 73,44 & 64,11 & 78,75 & 111,11 \\
\hline 2 & 6,44 & 389,11 & 66,00 & 133,44 & 17,33 & 67,50 & 5 & 41,78 & 27,29 & 73,78 & 114,22 & 6,89 \\
\hline 3 & 01,25 & 190,86 & 127,57 & 172,75 & 85,67 & 41,67 & 247,89 & 48,25 & 45,75 & 85,22 & 88,00 & 75,33 \\
\hline 4 & 4,00 & 86,71 & 78,75 & 408,78 & 66,57 & 106,43 & 88,25 & 251,13 & 110,14 & 132,50 & 146,83 & 106,14 \\
\hline 5 & 2,11 & 718,00 & 37,00 & 509,11 & 126,88 & 165,25 & $11 \varepsilon$ & 108,22 & 13 & & 33 &, 56 \\
\hline$\epsilon$ & 27,33 & 173,50 & 03,60 & 204,50 & 120,17 & 184,00 & 115,29 & 146,00 & 174,60 & 153,86 & 71,17 & 83,00 \\
\hline 7 & 39,20 & 269,63 & 93,75 & 266,25 & 209,86 & ,89 & 179,71 & 911,75 & 00 & 208,38 & 1,43 & 299,29 \\
\hline 8 & 1,75 & 188,00 & 213,44 & 451,13 & 136,63 & 135,43 & 207,80 & 152,33 & 112,00 & 115,50 & 36,44 & 231,89 \\
\hline s &, 33 & 276,67 & 176,29 & 117,88 & 161,80 & 90,00 & 140,00 & 204,88 & 101,56 & 196,17 & 143,78 & 148,00 \\
\hline 1 & 7,63 & 305,78 & 11,33 & 141,00 & 240,22 & 355,56 & 293,89 & 282,22 & 289,75 & 240,86 & 274,89 & 226,33 \\
\hline 1 & 0,56 & 264,44 & 69,63 & 162,29 & 173,75 & 127,17 & 172,89 & 148,89 & 107,67 & 95,75 & 13,00 & 167,00 \\
\hline 1 & 5,88 & 659,50 & 78,38 & 421,33 & 39,00 & 196,11 & 70,00 & 201,89 & 181,33 & 96,44 & 107,22 & 59,78 \\
\hline 1 & 9,00 & 76,60 & 251,71 & 234,00 & 180,13 & 212,00 & 171,80 & 115,00 & 67,25 & 102,00 & 111,50 & 183,13 \\
\hline 14 & 113,00 & 104,43 & 159,00 & 103,00 & 142,00 & 186,78 & 75,67 & 176,25 & 116,33 & 124,75 & 8,67 & 101,56 \\
\hline 15 & 63, & 83,78 & 72,43 & 116,29 & 86 & 112,50 & 110,00 & 80,25 & 4,60 & 70,67 & 5,00 & 80,56 \\
\hline 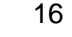 & 57,50 & 74,00 & 78,17 & 120,86 & 130,38 & 49,50 & 54,89 & 56,29 & 55,00 & 73,33 & 42,75 & 45,13 \\
\hline 17 & 235,22 & 185,25 & 134,20 & 155,29 & 91,13 & 157,00 & 93,38 & 72,11 & 73,38 & 59,38 & 83,00 & 85,33 \\
\hline 1 & 5,43 & 77,00 & 69,71 & 81,50 & 112,40 & 91,22 & 129,11 & 143,88 & 149,00 & 43,25 & 583,60 & 102,13 \\
\hline & & 114,50 & 16,50 & 75,50 & 11 & 102,11 & 64,33 & ,88 & ,56 & 95,89 & 9,00 & 151,33 \\
\hline 20 & 172,80 & 86,78 & 90,44 & 196,25 & 78,56 & 80,56 & 142,44 & 158,88 & 111,86 & 99,71 & 83,22 & 86,78 \\
\hline
\end{tabular}


ANEXO I - continuação.

\section{Erro variável}

\begin{tabular}{|c|c|c|c|c|c|c|c|c|c|c|c|c|}
\hline \multicolumn{13}{|l|}{$\begin{array}{c}\text { Sujeito } \\
\text { CO }\end{array}$} \\
\hline 1 & 211,64 & 35,70 & 54,92 & 199,62 & 438,84 & 172,85 & 463,22 & 281,24 & 472,00 & 463,02 & 617,13 & 223,66 \\
\hline 2 & 109,47 & 198,32 & 38,71 & 175,22 & 75,01 & 94,31 & 101,85 & 40,89 & 103,69 & 133,65 & 96,63 & 56,09 \\
\hline 3 & 764,09 & 178,42 & 204,21 & 227,43 & 129,56 & 144,69 & 110,86 & 139,22 & 258,72 & 214,44 & 179,48 & 237,12 \\
\hline 4 & 101,35 & 97,54 & 341,65 & 133,28 & 110,65 & 57,97 & 64,56 & 103,55 & 10,21 & 299,34 & 257,26 & 179,57 \\
\hline 5 & 293,70 & 78,68 & 40,20 & 72,49 & 92,44 & 120,84 & 87,53 & 125,60 & 217,43 & 126,28 & 96,20 & 106,07 \\
\hline 6 & 177,51 & 110,19 & 47,00 & 109,94 & 88,73 & 238,15 & 51,49 & 97,21 & 193,23 & 190,12 & 85,48 & 95,39 \\
\hline 7 & 85,05 & 148,97 & 95,31 & 51,68 & 51,19 & 71,53 & 91,53 & 86,20 & 55,47 & 81,72 & 89,81 & 203,53 \\
\hline 8 & 1157,06 & 37,42 & 67,88 & 36,12 & 48,62 & 59,40 & 254,67 & 120,14 & 128,85 & 84,33 & 157,18 & 166,58 \\
\hline 9 & 329,50 & 166,82 & 131,78 & 241,57 & 82,86 & 71,26 & 84,07 & 85,16 & 160,01 & 199,39 & 132,93 & 329,57 \\
\hline 10 & 146,62 & 114,64 & 303,84 & 249,07 & 296,12 & 172,79 & 247,75 & 345,37 & 928,98 & 229,76 & 89,85 & 222,66 \\
\hline 11 & 217,74 & 155,26 & 110,58 & 84,80 & 69,75 & 223,61 & 112,95 & 73,21 & 62,35 & 133,49 & 161,97 & 404,52 \\
\hline 12 & 147,25 & 144,13 & 158,95 & 127,63 & 262,58 & 213,32 & 130,16 & 118,73 & 131,65 & 227,74 & 99,81 & 82,62 \\
\hline 13 & 276,39 & 125,57 & 68,66 & 56,45 & 83,37 & 65,66 & 74,76 & 150,64 & 89,65 & 60,25 & 130,57 & 157,72 \\
\hline 14 & 83,27 & 96,47 & 56,33 & 152,61 & 54,08 & 88,09 & 167,91 & 180,14 & 193,07 & 184,10 & 106,48 & 106,24 \\
\hline 15 & 407,22 & 206,81 & 128,78 & 71,21 & 112,77 & 100,39 & 55,63 & 105,79 & 160,87 & 118,99 & 359,78 & 451,75 \\
\hline 16 & 85,77 & 50,91 & 117,82 & 72,83 & 192,74 & 329,67 & 145,72 & 264,93 & 68 & 172,78 & 06,80 & 95,12 \\
\hline 17 & 125,09 & 351,36 & 99,24 & 104,49 & 113,29 & 127,78 & 84,61 & 314,27 & 74,55 & 239,37 & 261,46 & 274,72 \\
\hline 18 & 245,15 & 47,12 & 145,31 & 67,18 & 167,00 & 58,37 & 94,04 & 173,84 & 138,77 & 80,39 & 106,67 & 80,03 \\
\hline 19 & 70,82 & 102,37 & 62,49 & 87,33 & 69,69 & 109,41 & 0,72 & 76,98 & 87,50 & 219,12 & 24,89 & 107,16 \\
\hline 20 & 255,72 & 73,85 & 219,88 & 149,21 & 87,91 & 78,60 & 87,23 & 40,43 & 67,14 & 166,61 & 187,01 & 77,80 \\
\hline \multicolumn{13}{|l|}{$A L$} \\
\hline 1 & 189,34 & 137,23 & 125,61 & 429,44 & 172,71 & 69,20 & 169,77 & 207,96 & 284,70 & 67,92 & 378,75 & 185,73 \\
\hline 2 & 112,92 & 56,46 & 53,84 & 70,25 & 107,94 & 102,18 & 108,96 & 43,58 & 46,79 & 91,05 & 58,12 & 63,92 \\
\hline 3 & 261,48 & 644,52 & 236,34 & 688,89 & 419,89 & 541,30 & 581,00 & 195,01 & 245,66 & 230,47 & 430,95 & 323,57 \\
\hline 4 & 309,00 & 297,24 & 129,49 & 99,18 & 156,53 & 26,01 & 48,20 & 33,27 & 52,18 & 241,94 & 230,99 & 63,47 \\
\hline 5 & 124,48 & 134,24 & 129,22 & 80,59 & 38,47 & 83,52 & 131,86 & 33,90 & 70,08 & 40,09 & 49,64 & 59,34 \\
\hline 6 & 45,84 & 62,63 & 157,75 & 56,38 & 117,38 & 43,21 & 64,99 & 64,30 & 47,47 & 180,24 & 32,75 & 34,63 \\
\hline 7 & 152,16 & 265,40 & 195,67 & 197,57 & 894,39 & 443,69 & 404,12 & 136,19 & 207,66 & 223,05 & 91,22 & 149,93 \\
\hline 8 & 114,07 & 115,66 & 118,02 & 351,45 & 560,50 & 114,04 & 389,82 & 334,48 & 53,27 & 82,43 & 137,80 & 64,04 \\
\hline 9 & 64,23 & 94,64 & 90,24 & 44,84 & 94,48 & 57,81 & 51,26 & 38,26 & 48,93 & 40,96 & 61,93 & 68,06 \\
\hline 10 & 650,78 & 382,91 & 312,09 & 411,73 & 230,87 & 215,99 & 450,94 & 171,91 & 135,18 & 133,62 & 261,59 & 183,06 \\
\hline 11 & 429,93 & 60,43 & 135,65 & 79,14 & 88,77 & 77,84 & 73,74 & 87,72 & 84,84 & 108,35 & 74,29 & 121,65 \\
\hline 12 & 1024,83 & 592,85 & 281,08 & 470,34 & 392,24 & 535,78 & 119,21 & 239,88 & 179,08 & 66,13 & 115,18 & 175,96 \\
\hline 13 & 387,53 & 1137,55 & 379,57 & 187,37 & 280,54 & 164,46 & 67,00 & 559,89 & 558,37 & 207,80 & 154,89 & 144,05 \\
\hline 14 & 769,58 & 431,61 & 308,68 & 381,04 & 190,52 & 173,02 & 207,89 & 278,66 & 191,45 & 115,44 & 195,18 & 63,68 \\
\hline 15 & 872,94 & 318,93 & 230,68 & 125,32 & 165,24 & 127,93 & 90,80 & 74,05 & 258,22 & 98,51 & 158,42 & 186,51 \\
\hline 16 & 279,60 & 74,64 & 61,01 & 72,59 & 106,60 & 95,89 & 88,86 & 211,77 & 144,68 & 61,67 & 82,40 & 62,79 \\
\hline 17 & 746,00 & 924,80 & 1159,60 & 642,37 & 452,47 & 579,83 & 464,76 & 387,87 & 981,35 & 193,53 & 246,70 & 310,99 \\
\hline 18 & 366,36 & 196,93 & 185,69 & 172,11 & 204,56 & 391,81 & 249,94 & 191,36 & 205,48 & 458,57 & 231,30 & 136,80 \\
\hline 19 & 541,43 & 865,58 & 299,82 & 252,65 & 519,39 & 132,06 & 211,51 & 239,12 & 531,40 & 158,47 & 98,31 & 181,32 \\
\hline 20 & 839,22 & 356,84 & 226,83 & 291,21 & 194,20 & 231,19 & 380,48 & 92,75 & 131,52 & 116,03 & 80,98 & 73,92 \\
\hline
\end{tabular}


ANEXO I - continuação.

CO-AL

\begin{tabular}{|c|c|c|c|c|c|c|c|c|c|c|c|c|}
\hline & 9,73 & 79 & 6,85 & 43,26 & 141,68 & 250,26 & 0,38 & ,85 & 10 & 31 & 20 & \\
\hline 2 & 8,56 & 27,50 & 9,47 & 61,10 & 99,68 & 122,18 & 88,19 & 237,26 & 213,55 & 8435 & 3,79 & 1,55 \\
\hline & 0,61 &, 92 & 8,64 & 65,42 & ,90 & 65 & 7,50 & 9,55 & 73 & 97 & 49 & 4,88 \\
\hline & 8,53 & 08,11 & 3,91 & 71,06 & 55,47 & 86,97 & 06,37 & 59,85 & 123,39 & & 90 & 4,29 \\
\hline & 43,70 & 70,63 & 3,67 & 78,58 & 69,15 & , 51 & 52,92 & 23,12 & 98,48 & 7,47 & 8,58 & 1,92 \\
\hline & 3,74 & 76,30 & 8,66 & ,29 & 90 & 19 & 1,07 & 47 & 40 & 25 & 74 & 7,24 \\
\hline & 5,48 & 18,20 & 0,07 & 198,30 & 23,17 & 4,76 & 11,93 & 29,73 & 354,40 & 16,45 & 70 & 2,73 \\
\hline & 55,16 & 39,83 & 6,59 & 118,00 & 113,51 & 140,72 & 97,71 & 54,27 & 66,84 & 16,00 & 6,65 & 2,81 \\
\hline & 4,46 & 62,86 & 4,47 & 103,86 & 4,74 & 309,93 & 37,77 & 7,40 & 77,31 & 33 & 6,71 & 18,72 \\
\hline & 3,12 & 250,95 & 96,53 & 54,68 & 170,13 & 270,53 & 329,03 & 14,60 & 368,67 & 274,40 & 3,53 & 78,46 \\
\hline & 7,57 & 119,71 & 85,53 & 82,87 & 217,62 & 1,39 & 35,05 & 73,14 & 183,77 & 105,30 & 54,76 & 277,45 \\
\hline & 6,56 & 50,98 & 87,78 & 88,99 & 91,16 & 273,44 & 95,25 & 7,18 & ,01 & 92,36 & 1,89 & 6,93 \\
\hline & 4,88 & 50,94 & 70,63 & 85,95 & 344,40 & & 7,10 & 3,44 & 89 & ,32 & 27 & 1,25 \\
\hline & 0,52 & 68,06 & 46,83 & 62,72 & 130,63 & & 32,64 & 77,77 & 33 & 72,03 & 21 & 3,99 \\
\hline & 5,66 & 5,62 & 45,42 & 96,50 & 374,96 & ,27 & 97,96 & 55,44 & 19 & 8 & 62 & 2,72 \\
\hline & 3,29 & 2 & 5,85 & 2 & 57,97 & 4 &, 82 & 1 & 41 & 5 & &, 48 \\
\hline & 4,82 & 43,22 & 25,09 & 134,27 & 34,28 & 70 & 63,57 & 37,55 & 47,04 & 73,89 & 58 & 1,05 \\
\hline & 2,51 & 47,46 & 3,14 & 214,86 & 257,58 & 158,66 & 222,25 & 1,44 & 0,38 & 157,42 & 0,34 & 38,21 \\
\hline & 9,15 & 157,89 & 0,83 & 158,84 & 726,49 & 2,53 & 43,68 & 8,46 & 42,41 & $4 S$ & 92 & 57,82 \\
\hline & 1,18 & 75.27 & 0 & 94.83 & 6 & 91 & 52,21 & 0,22 & 94,14 & 61,84 & 02 & 7,02 \\
\hline \\
\hline & 71 & 0,32 & ,93 & 48,56 & 29 &, 21 & 47 & 70,96 & 18,41 & 6 & 1,15 & 5,80 \\
\hline & 5,14 & 412,44 & 0,33 & 161,22 & 149,29 & 6,92 & 46,37 & 48,32 & 123,74 & 1,59 & 01,20 & 124,64 \\
\hline & & 98 & 9 & 1 & 12 & & 49 & 55 & & & & ,33 \\
\hline & 1,75 & 54,99 & ;,39 & 768,84 & 54,88 & 4,73 & 77,83 & 226,44 & 6,73 & 194,43 & 39 & 92,09 \\
\hline & , 92 & 223,54 & 5,43 & 63,74 & 33,95 & 44 & 94,87 & 14 & 74 & 79 & 47 & 112,51 \\
\hline 6 & 57 & 137,89 & 8,27 & 52,40 & 1 & & 6,37 & 34 & 52 & & &, 40 \\
\hline & 3,82 & 59 & 2,22 & 340,03 &, 13 & 35 & 59,74 & 912,24 & 48,09 & 174,21 & 57 & 350,52 \\
\hline & 38 & 19 & 25 & 39 & 6 & 99 & 83 & 36 & 97 & 50 & 08 & 7,26 \\
\hline & & 3,23 & 4,80 & 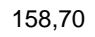 & & & 39 & 57 & 104,54 & & & 185,66 \\
\hline & $\hat{5}, 41$ & 9,71 & 8,96 & 192,26 & 30,58 &, 54 & 0,74 & 237,89 &, 48 & 255,38 & 6,94 & 3,63 \\
\hline & 3,68 & 6,54 & 7,75 & 4,54 & ,12 & 71 & 11,51 & , 09 & 55 & 132,87 & 19 & 204,12 \\
\hline 1 & 4,67 & 551,11 & 6,46 & 54,94 & 8,00 & 48 & 75,48 & 3,43 & 407,65 & 117,94 & 87 & 6,56 \\
\hline 1 & & & 58 & 2,07 & & 39 & 0,45 &, 71 & 79 & & 63 & 2,85 \\
\hline 1 & 3,66 & 33,58 & 24,86 & 127 & 168 & & 86,46 & 2,16 & 131,27 & 135,92 & & 126,45 \\
\hline 15 & 146,79 & 125,40 & 3,46 & 136,50 & 105 & 124,24 & 109,95 & 118,40 &, 19 & 08 & 102,45 & 124,16 \\
\hline 16 & & & 31 & 12 & 148 & & 77,70 & ,76 & 03 & 7 & 06 & 2,61 \\
\hline 1 & & & 36 & 180 & & & & & & & & \\
\hline IC & & & & & & & 32 & & &, 59 &, 19 & 3,41 \\
\hline 19 & 1,42 & 103,69 & 31,14 & 120,55 & 18 & ,33 & 97,24 &, 06 & 120,76 & 104,57 & 5,48 & 185,67 \\
\hline & & & & 8.13 & 2,99 & 6.45 & 9.74 & 5,12 & 127.46 & 22,00 & 07.67 & \\
\hline
\end{tabular}


ANEXO I - continuação.

\section{Erro constante}

\begin{tabular}{|c|c|c|c|c|c|c|c|c|c|c|c|c|}
\hline $\begin{array}{c}\text { Sujeito } \\
\text { CO }\end{array}$ & \multicolumn{11}{|c|}{$\mathrm{CO}$} & A4 \\
\hline 1 & 214,00 & 71,50 & 24,88 & 141,75 & 497,38 & 427,75 & 518,25 & 565,78 & 493,63 & 580,11 & 839,43 & 322,57 \\
\hline 2 & $-45,60$ & 64,75 & 8,33 & $-8,00$ & $-13,22$ & 12,33 & $-70,00$ & $-73,25$ & $-15,00$ & $-15,25$ & $-57,00$ & $-45,50$ \\
\hline 3 & 1133,63 & 106,00 & 102,71 & 242,33 & 125,43 & 108,25 & 83,56 & 160,00 & 191,75 & 155,11 & 176,50 & 320,14 \\
\hline 4 & $-134,00$ & $-39,88$ & 160,40 & 31,50 & 90,80 & $-49,00$ & $-1,00$ & $-90,33$ & 72,67 & 297,75 & 166,75 & 112,17 \\
\hline 5 & $-36,67$ & $-71,80$ & $-57,50$ & $-46,86$ & $-72,67$ & $-70,33$ & $-13,63$ & $-41,86$ & $-25,17$ & $-111,57$ & $-76,00$ & $-235,00$ \\
\hline 6 & 66,75 & 213,00 & 36,71 & 80,83 & $-64,40$ & 137,86 & 2,67 & $-62,00$ & 202,60 & 52,14 & $-83,00$ & $-61,50$ \\
\hline 7 & $-26,50$ & 23,25 & 52,38 & 55,88 & 65,75 & 30,25 & 96,50 & 96,67 & 63,78 & 56,00 & 40,89 & 156,13 \\
\hline 8 & 506,67 & $-191,33$ & 78,00 & $-3,33$ & $-0,60$ & $-117,86$ & $-78,50$ & $-149,29$ & $-30,83$ & 107,33 & $-20,50$ & $-52,17$ \\
\hline 9 & $-138,88$ & $-236,78$ & $-192,89$ & 89,63 & $-10,78$ & $-31,75$ & 7,00 & $-168,00$ & $-77,43$ & 10,75 & $-42,88$ & 63,60 \\
\hline 10 & $-26,40$ & 47,67 & 343,17 & 162,40 & 147,00 & 161,00 & 141,88 & 338,50 & 556,71 & 123,29 & 21,33 & $-29,29$ \\
\hline 11 & 43,00 & 25,75 & 0,00 & $-62,43$ & $-50,38$ & 64,00 & 19,78 & $-62,44$ & $-65,11$ & $-15,00$ & $-4,44$ & 129,11 \\
\hline 12 & $-121,20$ & 9,14 & $-157,00$ & 17,89 & 44,22 & $-21,67$ & $-23,13$ & $-49,75$ & $-50,57$ & 45,50 & 6,33 & 58,89 \\
\hline 13 & 30,13 & $-79,25$ & $-57,88$ & 23,43 & $-49,78$ & $-83,67$ & $-50,50$ & $-9,89$ & $-26,13$ & 89,00 & 17,50 & $-10,44$ \\
\hline 14 & $-81,63$ & $-86,00$ & $-72,00$ & $-65,75$ & $-27,67$ & $-36,50$ & $-128,80$ & $-13,43$ & $-33,89$ & $-90,25$ & $-89,67$ & $-136,57$ \\
\hline 15 & 263,43 & 81,56 & 40,00 & $-16,00$ & $-43,44$ & 35,22 & 14,86 & 30,00 & 155,89 & 133,11 & 268,50 & 317,50 \\
\hline 16 & $-81,75$ & $-43,00$ & $-12,67$ & $-25,50$ & 36,44 & 238,00 & 11,33 & 196,00 & $-1,75$ & 79,17 & 76,56 & 323,57 \\
\hline 17 & $-113,40$ & $-24,29$ & $-113,22$ & $-85,78$ & $-85,00$ & $-67,75$ & $-41,38$ & 146,33 & 44,00 & 254,80 & 241,00 & 286,71 \\
\hline 18 & $-124,00$ & $-25,50$ & $-66,56$ & $-19,25$ & $-24,25$ & $-14,00$ & $-48,75$ & $-10,44$ & 69,00 & 94,33 & 49,14 & $-25,00$ \\
\hline 19 & $-42,50$ & $-80,13$ & 6,75 & $-44,33$ & $-21,56$ & $-73,56$ & $-40,13$ & $-36,67$ & $-72,00$ & $-6,88$ & $-52,00$ & $-3,57$ \\
\hline 20 & $-100,00$ & 1,29 & $-149,80$ & $-87,00$ & $-28,00$ & $-33,11$ & $-44,44$ & $-39,63$ & 16,25 & 8,25 & 45,63 & $-74,11$ \\
\hline \multicolumn{13}{|l|}{$A L$} \\
\hline 1 & 143,00 & 280,57 & 96,38 & 288,25 & 30,11 & 17,25 & 91,63 & 128,86 & 56,00 & $-22,56$ & 281,11 & 40,00 \\
\hline 2 & $-59,50$ & $-83,83$ & $-95,63$ & $-86,78$ & $-82,00$ & $-54,44$ & $-136,50$ & $-30,86$ & $-33,67$ & $-72,14$ & $-68,33$ & $-55,63$ \\
\hline 3 & 446,00 & 550,33 & 612,33 & 805,40 & 699,60 & 685,67 & 711,17 & 780,50 & 414,83 & 998,40 & 952,83 & 821,29 \\
\hline 4 & 18,13 & 58,29 & $-124,33$ & $-50,75$ & $-82,50$ & $-50,17$ & $-24,67$ & $-61,38$ & $-53,88$ & 22,13 & $-108,56$ & 1,13 \\
\hline 5 & $-330,00$ & $-239,50$ & $-204,44$ & $-54,80$ & $-50,57$ & $-7,14$ & $-79,50$ & $-50,00$ & $-10,00$ & $-24,43$ & $-42,83$ & $-29,86$ \\
\hline 6 & 28,00 & $-48,38$ & 3,86 & $-64,50$ & $-53,25$ & $-26,00$ & $-66,63$ & $-67,71$ & $-55,44$ & $-58,67$ & $-36,43$ & $-59,88$ \\
\hline 7 & 108,43 & 155,67 & 106,29 & 110,40 & 1057,33 & 492,44 & 283,44 & 100,13 & 111,89 & 170,56 & $-10,14$ & 52,67 \\
\hline 8 & $-7,00$ & 123,00 & 235,80 & 151,00 & 289,60 & $-57,00$ & 182,25 & 112,00 & $-90,83$ & 27,67 & $-12,71$ & $-56,75$ \\
\hline 9 & $-42,56$ & $-6,17$ & $-60,25$ & $-61,00$ & $-64,88$ & $-66,75$ & $-83,67$ & $-87,17$ & $-79,78$ & $-106,22$ & $-95,44$ & $-109,33$ \\
\hline 10 & 974,50 & 313,75 & 520,13 & 421,17 & 301,63 & 549,57 & 356,88 & 52,14 & 129,14 & $-37,11$ & 43,29 & $-50,83$ \\
\hline 11 & $-86,50$ & $-77,29$ & $-71,88$ & $-63,44$ & $-80,50$ & $-67,22$ & $-74,13$ & $-126,89$ & $-60,38$ & $-89,11$ & $-140,83$ & $-133,00$ \\
\hline 12 & 1170,00 & 144,83 & $-22,63$ & 291,22 & 59,88 & 118,44 & $-124,13$ & $-2,67$ & 13,14 & $-141,40$ & $-28,57$ & 166,63 \\
\hline 13 & 229,67 & 531,33 & 254,22 & 90,00 & 35,33 & 4,33 & $-67,29$ & 261,50 & 250,83 & 27,56 & $-121,86$ & 11,33 \\
\hline 14 & 269,50 & 275,50 & 4,67 & $-2,80$ & $-132,11$ & $-259,00$ & $-85,13$ & $-8,00$ & $-39,38$ & $-84,56$ & $-129,00$ & $-148,89$ \\
\hline 15 & 692,38 & 94,89 & 50,44 & 11,89 & 130,63 & 52,14 & $-6,56$ & 16,50 & 90,33 & $-42,67$ & 11,75 & 69,00 \\
\hline 16 & 65,22 & $-60,89$ & $-33,50$ & $-37,50$ & $-78,75$ & $-42,11$ & 2,50 & 3,75 & $-12,56$ & $-70,75$ & $-39,75$ & $-48,89$ \\
\hline 17 & 797,50 & 1052,17 & 719,50 & 442,13 & 436,88 & 359,50 & 430,80 & 339,25 & 891,00 & 229,57 & 232,71 & 297,50 \\
\hline 18 & 318,63 & 136,33 & $-34,43$ & 7,00 & $-65,00$ & 111,40 & 75,50 & 193,38 & 158,89 & 236,13 & $-1,89$ & $-53,44$ \\
\hline 19 & 525,00 & 307,56 & $-24,78$ & 63,25 & 31,00 & $-42,44$ & 7,43 & 106,14 & 5,13 & $-79,25$ & $-27,11$ & $-227,38$ \\
\hline 20 & 840,14 & 672,22 & 513,75 & 400,86 & 275,75 & 261,00 & 219,57 & 167,89 & 151,00 & 70,00 & $-10,56$ & $-80,67$ \\
\hline
\end{tabular}


ANEXO I - continuação.

CO-AL

\begin{tabular}{|c|c|c|c|c|c|c|c|c|c|c|c|c|}
\hline 1 & 4,50 & 61,44 & 30,75 & 103,44 & 73,43 & 121,44 & $-14,00$ & 44,57 & 70,38 & 66,43 & 68,78 & \\
\hline 2 & 49,00 & 111,25 & 4,00 & $-2,00$ & 84,43 & 55,56 & 26,86 & 125,25 & 99,17 & 67,75 & $-15,63$ & $-20,13$ \\
\hline 3 & $-76,67$ & $-42,67$ & $-147,44$ & $-96,00$ & 25,50 & 329,29 & $-27,29$ & 29,17 & 5,00 & 160,17 & $-12,57$ & $-41,86$ \\
\hline 4 & $-124,33$ & 334,43 & 3,13 & $-55,00$ & $-38,13$ & $-3,17$ & 8,67 & 6,67 & 65,83 & $-46,29$ & 14,13 & $-7,14$ \\
\hline 5 & $-124,86$ & $-125,80$ & $-16,43$ & $-43,25$ & $-11,00$ & 5,80 & 119,33 & $-94,33$ & $-10,67$ & $-10,25$ & 7,88 & 15,22 \\
\hline 6 & $-127,22$ & $-99,14$ & $-114,25$ & $-181,50$ & $-122,78$ & $-157,00$ & $-230,43$ & $-249,71$ & $-269,14$ & $-61,67$ & $-165,38$ & $-56,67$ \\
\hline 7 & 595,75 & $-93,75$ & 74,33 & $-120,67$ & $-19,33$ & 310,11 & 504,29 & 193,86 & 46,17 & $-70,71$ & $-173,88$ & $-207,57$ \\
\hline 8 & $-84,33$ & $-59,40$ & 4,50 & $-115,00$ & $-127,78$ & $-139,00$ & $-135,56$ & $-114,38$ & $-188,22$ & $-183,38$ & $-162,63$ & $-150,89$ \\
\hline 9 & $-166,13$ & $-40,22$ & $-22,33$ & $-17,67$ & 354,80 & 403,00 & 297,71 & $-87,38$ & 102,22 & 0,50 & 46,43 & 85,00 \\
\hline 10 & $-355,00$ & $-232,80$ & $-281,75$ & 21,33 & $-129,60$ & 278,50 & 79,89 & 234,50 & 243,14 & 174,56 & $-112,57$ & 92,83 \\
\hline 11 & $-31,71$ & $-27,14$ & $-48,67$ & $-63,25$ & $-22,63$ & $-19,00$ & $-45,50$ & $-30,88$ & $-43,63$ & $-113,33$ & $-57,75$ & $-18,14$ \\
\hline 12 & $-42,13$ & $-13,38$ & $-29,00$ & $-81,75$ & $-47,25$ & 51,00 & $-13,88$ & $-55,50$ & $-31,13$ & $-75,13$ & $-59,67$ & $-71,11$ \\
\hline 13 & 215,22 & 20,00 & 19,00 & $-13,78$ & 135,57 & 142,57 & 57,20 & 105,25 & $-9,00$ & $-14,89$ & $-51,67$ & $-44,00$ \\
\hline 14 & 36,14 & $-65,22$ & $-59,25$ & $-28,38$ & $-103,83$ & $-17,00$ & $-74,57$ & $-63,50$ & $-96,25$ & $-47,13$ & $-63,67$ & $-42,44$ \\
\hline 15 & $-228,71$ & $-138,78$ & $-22,89$ & $-28,67$ & 137,13 & $-19,13$ & 20,78 & $-22,00$ & $-45,11$ & $-29,89$ & $-59,88$ & $-69,33$ \\
\hline 16 & 927,67 & 552,13 & $-2,38$ & $-6,63$ & 531,57 & 347,78 & $-68,11$ & 17,50 & $-123,71$ & 1,56 & 189,38 & $-35,00$ \\
\hline 17 & $-21,22$ & $-48,75$ & $-90,00$ & $-78,25$ & 22,00 & 13,71 & 17,22 & $-30,00$ & 16,63 & 20,00 & $-8,38$ & $-7,33$ \\
\hline 18 & $-56,20$ & $-15,71$ & $-1,75$ & 81,67 & 208,57 & 108,88 & 93,14 & 136,20 & 115,78 & 90,00 & 88,75 & 32,67 \\
\hline 19 & $-1,20$ & 13,57 & $-9,88$ & $-132,00$ & 441,20 & 21,00 & 166,86 & 61,00 & $-43,14$ & $-25,20$ & $-38,50$ & 108,00 \\
\hline 20 & 204,86 & 53,78 & $-114,11$ & $-10,50$ & 2,43 & $-5,75$ & $-54,78$ & 72,56 & 204,44 & $-75,89$ & $-272,33$ & $-169,25$ \\
\hline \multicolumn{13}{|l|}{ L-CC } \\
\hline 1 & 376,50 & 175,13 & 196,14 & 97,11 & 82,44 & 57,88 & 66,89 & 83,13 & 73,44 & 35,22 & 78,75 & 111,11 \\
\hline 2 & 916,44 & 376,00 & 249,75 & 98,33 & 79,78 & $-22,75$ & 0,25 & $-23,78$ & 120,14 & 9,11 & 50,22 & 11,78 \\
\hline 3 & 201,25 & 190,00 & 122,43 & 172,75 & 21,89 & 41,67 & 225,22 & 28,75 & 42,50 & 85,22 & 84,00 & 75,33 \\
\hline 4 & $-113,50$ & 18,71 & $-55,75$ & 337,67 & $-55,43$ & $-91,57$ & $-65,25$ & $-251,13$ & $-110,14$ & 3,17 & $-146,83$ & $-106,14$ \\
\hline 5 & 530,11 & 718,00 & 546,25 & 414,00 & 84,38 & $-86,25$ & $-97,25$ & $-65,56$ & 162,88 & $-59,00$ & $-27,78$ & 29,33 \\
\hline 6 & 85,33 & 173,50 & 196,40 & 188,75 & 120,17 & 148,00 & 96,14 & 109,43 & 174,60 & 153,86 & $-63,50$ & $-83,00$ \\
\hline 7 & $-2,80$ & $-269,63$ & 49,75 & 9,50 & $-209,86$ & $-20,44$ & 82,29 & 836,75 & 114,57 & $-137,13$ & 179,71 & 119,86 \\
\hline 8 & 47,75 & 116,50 & 201,00 & 435,63 & $-9,88$ & $-29,43$ & $-49,40$ & $-152,33$ & 1,14 & $-31,25$ & $-126,67$ & $-125,22$ \\
\hline 9 & $-92,00$ & 223,33 & $-1,14$ & $-37,88$ & $-161,80$ & $-60,25$ & 8,00 & $-204,88$ & $-66,00$ & $-113,83$ & $-122,89$ & $-90,50$ \\
\hline 10 & 657,63 & 195,11 & 264,44 & 15,67 & 116,44 & 312,22 & 126,78 & 188,89 & 289,75 & 117,43 & 51,56 & $-226,33$ \\
\hline 11 & 135,22 & 56,00 & 16,63 & $-25,43$ & $-47,25$ & $-125,83$ & 95,11 & $-110,44$ & $-48,33$ & $-52,25$ & 32,78 & $-68,33$ \\
\hline 12 & 685,88 & 659,50 & 428,13 & 230,67 & 136,71 & $-196,11$ & $-66,67$ & 26,11 & 107,78 & $-35,78$ & $-78,11$ & $-48,00$ \\
\hline 13 & 400,20 & 12,20 & $-251,71$ & 101,25 & 8,63 & $-182,00$ & $-171,80$ & $-113,29$ & $-67,25$ & $-102,00$ & $-63,00$ & $-113,88$ \\
\hline 14 & $-6,50$ & 2,71 & $-38,00$ & 14,43 & $-50,57$ & $-186,78$ & 23,00 & 101,75 & $-38,33$ & $-76,25$ & $-31,00$ & $-14,00$ \\
\hline 15 & $-50,38$ & $-9,11$ & $-24,71$ & $-84,29$ & $-34,71$ & $-112,50$ & $-99,25$ & 0,25 & $-23,00$ & $-16,00$ & $-60,00$ & 6,56 \\
\hline 16 & $-3,25$ & $-49,56$ & $-11,17$ & 94,86 & 23,88 & 44,00 & $-14,67$ & $-1,43$ & $-11,29$ & $-18,22$ & 15,00 & $-6,13$ \\
\hline 17 & 155,44 & $-3,25$ & $-111,00$ & $-37,00$ & $-49,88$ & $-86,25$ & $-71,38$ & $-65,00$ & $-37,38$ & $-53,88$ & $-14,50$ & 12,67 \\
\hline 18 & $-17,14$ & $-3,00$ & $-13,43$ & $-38,17$ & $-69,20$ & $-53,22$ & $-127,78$ & $-81,63$ & $-132,20$ & 6,75 & 540,80 & $-86,38$ \\
\hline 19 & 248,14 & 95,75 & 256,00 & $-24,75$ & $-40,33$ & $-59,00$ & $-17,44$ & $-20,38$ & 7,78 & $-51,89$ & $-32,33$ & 18,67 \\
\hline 20 & 158,40 & $-78,33$ & 25,33 & 196,25 & $-54,78$ & 10,78 & $-70,89$ & $-7,38$ & 93,57 & $-10,00$ & $-3,67$ & $-46,33$ \\
\hline
\end{tabular}


ANEXO II - Média dos erros absoluto, variável e constante, dos participantes dos grupos do experimento 1 (tarefa complexa), dos grupos CO, AL, CO-LA e AL-CO, dos oito blocos de tentativas da fase de estabilização (E1 a E8) e dos quatro blocos de tentativas da fase de adaptação (A1, A2, A3 e A4), em milissegundos.

\section{Erro Absoluto}

\begin{tabular}{|c|c|c|c|c|c|c|c|c|c|c|c|c|}
\hline $\begin{array}{l}\text { ujeito } \\
\text { CO }\end{array}$ & 1 & 2 & 3 & 4 & 5 & 6 & 7 & 8 & 1 & 2 & 3 & 4 \\
\hline 1 & 24,00 & 110,88 & 172,43 & 66,00 & 126,00 & 47,88 & 91,14 & 131,67 & 55,00 & 44,00 & 217,33 & 153,17 \\
\hline 2 & 468,33 & 587,67 & 278,00 & 280,50 & 195,14 & 171,80 & 287,57 & 09,15 & 594,00 & 267,60 & 72,60 & 4,00 \\
\hline 3 & 294,50 & 171,00 & 236,80 & 315,00 & 64,29 & 131,60 & 52,25 & 616,43 & 309,33 & 77,50 & 54,25 & 161,33 \\
\hline 4 & 647,83 & 283,00 & 329,00 & 352,00 & 161,00 & 165,20 & 346,33 & 500,80 & 694,67 & 112,00 & 138,60 & 280,29 \\
\hline 5 & 254,00 & 161,00 & 133,80 & 90,75 & 94,00 & 96,29 & 140,50 & 107,67 & 156,67 & 245,75 & 95,43 & 128,14 \\
\hline 6 & 486,40 & 589,25 & 320,86 & 260,71 & 287,86 & 118,71 & 88,50 & 60,67 & 462,33 & 417,57 & 168,75 & 62,67 \\
\hline 7 & 342,20 & 396,57 & 312,00 & 94,88 & 118,88 & 94,00 & 454,22 & 366,88 & 820,60 & 727,88 & 331,75 & 135,22 \\
\hline 8 & 533,57 & 1314,67 & 1082,50 & 736,78 & 481,56 & 501,25 & 293,50 & 293,44 & 1285,75 & 908,56 & 784,56 & 768,33 \\
\hline 9 & 150,33 & 35,75 & 128,38 & 123,11 & 46,50 & 125,00 & 142,88 & 185,17 & 329,71 & 214,43 & 80 & 237,29 \\
\hline 10 & 325,40 & 51,43 & 93,88 & 94,33 & 50,50 & 65,88 & 253,67 & 32,50 & 742,75 & 302,00 & 209,89 & 115,00 \\
\hline 11 & 177,78 & 210,00 & 212,80 & 73,86 & 97,13 & 68,50 & 39,63 & 85,63 & 162,00 & 103,00 & 140,63 & 52,50 \\
\hline I & $10<, 50$ & 112,50 & 49,00 & 101,00 & 87,43 & 113,33 & 103,86 & 127,00 & 283,83 & 78,00 & 4 & 225,25 \\
\hline 13 & 684,83 & 123,00 & 94,78 & 141,88 & 149,75 & דו & 93,78 & 148,86 & 208,50 & 103,75 & 143,78 & 178,75 \\
\hline 14 & 399,83 & 672,86 & 172,50 & 205,00 & 94,43 & 104,00 & 91,00 & 73,14 & 221,00 & 119,13 & 141,67 & 270,43 \\
\hline 15 & 561,60 & 237,63 & 173,00 & 69,20 & 130,25 & 89,75 & 113,50 & 91,25 & 211,50 & 162,00 & 82,17 & 135,33 \\
\hline 16 & 1472,00 & 360,25 & 2 & 1( & 0 & 81,89 & 00 & 67 &, 50 & 0 & 8 & 95,71 \\
\hline 17 & 421,67 & 455,80 & 513,00 & 373,44 & 314,17 & 362,88 & 396,33 & 458,86 & 431,17 & 627,71 & 446,25 & 411,14 \\
\hline 18 & 52,14 & 301,67 & 139 & 252,78 & 69,11 & 259,78 & 147,88 & 190,00 & 768,60 & 285,33 & & 134,00 \\
\hline 19 & , & 12 & 1 & 187 & 245,00 & 3 & 111,44 & 90 & 3 & 75 & 4 & 4 \\
\hline 20 & 07,00 & 101,86 & 40,63 & 122,43 & 98,38 & 143,44 & 182,89 & 79,67 & 465,67 & 346,25 & 102,11 & 174,00 \\
\hline \multicolumn{13}{|l|}{$A L$} \\
\hline 1 & 0 & 1 & 82,25 & 5 & 3 & 3 & 6 & 3 & 23 & 3 & 1 & 26,44 \\
\hline 2 & 18,00 & 304,25 & 170,33 & 214,75 & 140,25 & 200,33 & 82,29 & 68,67 & 64,67 & 100,50 & 172,57 & 49,50 \\
\hline 3 & 493,50 & 829,86 & 707,60 & 174,25 & 669,33 & 422,75 & 262,75 & 363,67 & 313,17 & 89,00 & 184,25 & 204,33 \\
\hline 4 & 234,00 & 267,57 & 404,40 & 169,57 & 106,75 & 249,38 & 77,29 & 135,57 & 136,33 & 105,75 & 1 & 81,00 \\
\hline 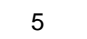 & 52,80 & 122,86 & 48,20 & 64,20 & 96,57 & שט, ות & 102,78 & 100,00 & 90,78 & 108,14 & & 100,00 \\
\hline 6 & 214,00 & 520,00 & 579,40 & 879,00 & 667,60 & 735,75 & 531,83 & 348,80 & 265,40 & 116,57 & 172,63 & 182,67 \\
\hline 7 & 95,33 & 162,13 & 539,13 & 442,14 & 138,50 & 248,78 & 227,50 & 119,86 & 182,14 & 131,75 & 106,78 & 82,38 \\
\hline 8 & 1305,00 & 309,00 & 150,00 & $8 / 4,00$ & 201,00 & $9<4,43$ & 401,33 & 287,13 & $1 / 2,00$ & 139,29 & & 429,63 \\
\hline 9 & 1826,00 & 581,00 & 1071,25 & 1319,17 & 494,33 & 256,00 & 153,13 & 173,89 & 450,00 & 206,13 & 168,25 & 148,50 \\
\hline 10 & 1321,43 & 836,00 & 719,25 & 219,89 & 192,00 & 133,33 & 147,33 & 213,56 & 174,56 & 172,44 & 78,22 & 64,11 \\
\hline 11 & 3 & 33 & 0 & 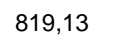 & 948,43 & 753,44 & 7 & & & & & 87 \\
\hline 10 & 8932 & 0767 & 111 & 72,00 & 147,00 & 176,89 & 82,50 & 55,44 & 13 & 44 & 82,44 & 98,89 \\
\hline 13 & 1681,00 & 765,67 & 937,75 & 1295,50 & 803,40 & 592,75 & 281,86 & 707,00 & 539,25 & 145,50 & 190,88 & 128,00 \\
\hline 14 & s & 1044,67 & 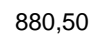 & s & 5 & 8 & 7 & U & 541,60 & U & 0 & 31 \\
\hline 15 & 492,50 & 659,57 & 541,63 & 462,11 & 205,56 & 174,56 & 87,22 & 334,00 & 184,38 & 150,33 & 179,00 & 107,22 \\
\hline 16 & 50,00 & 77,56 & 60,33 & 72,22 & 100,63 & 53,22 & 48,75 & 55,50 & 44, & 59,56 & 2 & 106,78 \\
\hline 17 & 280,67 & 268,00 & 403,50 & 291,01 & 131,01 & 127,00 & $7<, \angle 0$ & 94,50 & 93,14 & 05,80 & 49,89 & 85,67 \\
\hline 18 & 642,50 & 897,67 & 929,50 & 799,63 & 231,83 & 158,89 & 228,75 & 148,71 & 172,63 & 109,00 & 39 & 144,00 \\
\hline 19 & 04,17 & 997,60 & 89,17 & 444,67 & 552,83 & 307,75 & 145,14 & 218,14 & 626,00 & 94,63 & 268,83 & 89,43 \\
\hline 20 & 10,29 & 32,00 & 13,63 &, 57 & 07,63 & 81,75 & 736,29 & 1,33 & 1104,63 & 480,22 & ,22 & 249,22 \\
\hline
\end{tabular}


ANEXO II - continuação.

\begin{tabular}{|c|c|c|c|c|c|c|c|c|c|c|c|c|}
\hline \multicolumn{13}{|l|}{$\mathrm{O}-\mathrm{L}$} \\
\hline 1 & 89,75 & 46,60 & 103,29 & 57,11 & 163,33 & 148,00 & 43,43 & 60,57 & 65,80 & 38,33 & 50,00 & 84,57 \\
\hline 2 & 123,13 & 594,43 & 692,00 & 362,22 & 1691,71 & 1832,86 & 1573,67 & 1852,63 & 1233,89 & 776,44 & 307,67 & 263,13 \\
\hline 3 & 249,83 & 640,80 & 609,75 & 290,00 & 384,14 & 897,40 & 510,38 & 1083,00 & 1760,50 & 108,88 & 292,00 & 78,14 \\
\hline$T$ & 1039,00 & 340,50 & 558,67 & 346,78 & 1416,00 & 1040,00 & 737,60 & 696,50 & 752,71 & 771,00 & 545,25 & 51,25 \\
\hline 5 & 603,00 & 106,57 & 101,17 & 58,57 & 239,33 & 99,50 & 67,67 & 213,00 & 83,33 & 179,14 & 96,71 & 262,71 \\
\hline 6 & 317,50 & 81,67 & 68,75 & 60,67 & 248,33 & 55,50 & 111,00 & 188,29 & 453,17 & 66,80 & 92,67 & 63,40 \\
\hline 7 & & & 662,14 & 268,63 & & & & & 1065,00 & 425,44 & 353,67 & 246,88 \\
\hline 8 & 174,89 & 55,11 & 183,14 & 39,56 & 131,67 & 233,50 & 570,33 & 263,00 & 380,63 & 119,78 & 86,44 & 84,38 \\
\hline 9 & 527,50 & 186,56 & 426,78 & 97,22 & 495,50 & 156,75 & 198,57 & 227,00 & 200,00 & 88,00 & 89,67 & 101,33 \\
\hline 15 & 76,63 & 37,11 & 41,33 & 49,67 & 66,17 & 91,33 & 122,13 & 134,71 & 94,83 & 95,75 & 122,57 & 62,11 \\
\hline 11 & 10,80 & 5,50 & 59,00 & 160,11 & 111,86 & 72,29 & 1,33 & 6 & 4,00 & 46,29 & 00 & 99,33 \\
\hline 12 & 1461,33 & 1051,60 & 134,29 & 166,67 & 1946,50 & 1778,40 & 2569,40 & 1636,33 & 044,40 & 976,33 & 359,83 & 799,60 \\
\hline 13 & 181,75 & 172,14 & 83,50 & 70,00 & 364,33 & 29,14 & 106,25 & 380,33 & 391,40 & 135,63 & 177,75 & 63,33 \\
\hline 14 & 108,00 & 181,00 & 96,44 & 108,60 & 260,17 & 295,63 & 262,13 & 183,22 & 263,67 & 148,71 & 180,29 & 190,78 \\
\hline 15 & 309,67 & 194,50 & 110,78 & 163,11 & 194,00 & 253,33 & 229,40 & 151,17 & 115,33 & 126,22 & ,29 & 6,71 \\
\hline 16 & 126,00 & 185,00 & 166,00 & 201,00 & 50,00 & 310,57 & 146,80 & 329,14 & 76 & 16,33 & 76,00 & 128,67 \\
\hline 17 & 647,60 & 215,67 & 152,56 & 89,33 & 439,56 & 281,86 & 119,75 & 187,50 & 245,67 & 191,78 & 118,00 & 78,63 \\
\hline 1 & חم & 20 & 6 & 8 & 50 & 8 & 0 & 31,75 & 1. & 4 & 71 & $7 \mathrm{~S}$ \\
\hline 19 & 607,50 & 393,75 & 358,86 & 134,29 & 1050,50 & 1183,57 & 553,14 & 558,17 & 230,17 & 161,63 & 183,38 & 168,43 \\
\hline 20 & 620,44 & 24,00 & 0 & & 911,00 & 539,67 & & & & 53,00 & 83 & 266,50 \\
\hline \multicolumn{13}{|l|}{ L-CC } \\
\hline 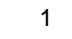 & 573,00 & 958,43 & 1053,50 & 705,00 & 555,17 & 235,00 & 188,14 & 170,88 & 325,83 & 300,43 & 221,88 & 178,71 \\
\hline 2 & 2076,80 & 1171,25 & 1225,50 & 1057,00 & 583,11 & 158,83 & 78,29 & 76,17 & 324,20 & 484,25 & 75,80 & 611,63 \\
\hline 3 & 1235,67 & 204,00 & 712,14 & 155,60 & 239,00 & 98,60 & 152,80 & 238,00 & 229,33 & 188,40 & 47,00 & 76,00 \\
\hline 4 & 299,50 & 28029 & 8686 & 180 & 10256 & G & 50 & 5 & 11 & 0 & 0 & 89,50 \\
\hline 5 & 486,71 & 451,75 & 528,75 & 151,44 & 333,67 & 505,33 & 448,60 & 329,00 & 130,67 & 199,56 & 122,11 & 229,22 \\
\hline 6 & 233,33 & 49,75 & 41,20 & 68,83 & 42,43 & 117,00 & 70,44 & 61,17 & 61,25 & 69,67 & 71,78 & 41,00 \\
\hline 7 & 5 & 0 & 7,83 & 46,80 & 3,00 & 110,33 & 106,63 & 40 & 33 & ,13 & 0 & 4,29 \\
\hline 8 & 54,17 & 652,75 & 375,29 & 277,13 & 161,43 & 73,13 & 69,67 & 124,13 & 109,20 & 127,50 & 101,50 & 143,50 \\
\hline 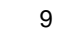 & 1047,67 & 1203,60 & 1581,89 & 1176,88 & 255,57 & 49,11 & 115,00 & 00,00 & 300,00 & 143,75 & 134,63 & 100,00 \\
\hline 10 & 42,38 & 134,89 & 646 & 654 & 39 & 3 & 55,11 & 47,13 & 0 & 3 & 1 & 11 \\
\hline 11 & 515,75 & 209,75 & 295,75 & 145,83 & 314,57 & 177,29 & 110,86 & 125,67 & 297,33 & 137,00 & 85,88 & 96,29 \\
\hline 12 & 838,33 & 1612,14 & 1191,20 & 845,17 & 755,20 & 360,43 & 608,29 & 524,44 & 1051,50 & 807,38 & 642,11 & 616,67 \\
\hline 13 & 14 & 23,56 & 43 & 224 & 100,22 & 18 & 67 & 33 & 83 & 5 & 13 & 4 \\
\hline 14 & 1852,75 & 1220,00 & 1282,67 & 1129,83 & 697,00 & 314,78 & 115,43 & 160,88 & 89 & 268,11 & 160,67 & 165,50 \\
\hline 15 & 914,11 & 724,78 & 635,33 & 472,67 & 493,44 & 179,33 & 528,22 & & & 56 & 357,33 & ז \\
\hline 16 & 176,00 & 1258,20 & 617,00 & 727,00 & 39,00 & 588,67 & 1,00 & 212,70 & 712,75 & 545,67 & 223,80 & $+10,00$ \\
\hline 17 & 1060,89 & 840,78 & 812,56 & 633,67 & 156,11 & 689,33 & 934,89 & 930,22 & 521,22 & 352,44 & 332,56 & 77,00 \\
\hline 18 & 785,25 & 782,40 & 239,40 & 159,00 & 868,00 & 184,00 & ,63 & 194,00 & 925,67 & 296,25 & 196,00 & 288,67 \\
\hline 19 & 100,00 & 63,00 & 122,29 & 137,17 & 30,50 & 46,43 & 54,88 & 40,00 & 127,00 & 331,57 & 101,00 & 91,78 \\
\hline 20 & 701,00 & 305,00 & 146,13 & 65,29 & 55,00 & 66,86 & 51,29 & 42,78 & 100,00 & 84,00 & 95,33 & 79,00 \\
\hline
\end{tabular}


ANEXO II - continuação.

\section{Erro Variável}

\begin{tabular}{|c|c|c|c|c|c|c|c|c|c|c|c|c|}
\hline ujeito & E1 & 2 & E3 & E4 & E5 & E6 & E7 & E8 & A1 & $\mathrm{A} 2$ & A3 & A4 \\
\hline \multicolumn{13}{|l|}{$\mathrm{CO}$} \\
\hline 1 & & 86 & 58.86 & 9251 & 57,86 & 58,84 & 49,92 & 143,40 & & 9,60 & 300,91 & 03,68 \\
\hline 2 & 85,29 & 22,83 & 121,03 & 203,33 & 31,12 & 105,74 & 353,12 & 86,46 & 633,03 & 79,61 & 17,54 & 37,03 \\
\hline 3 & 353,52 & 70,43 & 180,57 & 370,20 & 80 & 150,95 & 44 , & 784,43 & 37,49 & 7,69 & 02,93 & 0,00 \\
\hline 4 & 26,88 & 33,32 & 2,83 & 134,77 & 73,54 & 200,01 & 347,94 & 426,67 & 80,31 & 114,09 & 85,71 & 309,95 \\
\hline 5 & 258,94 & 223,62 & 113,58 & 122,81 & 137,72 & 124,55 & 198,70 & 119,30 & 228,22 & 303,97 & 140,92 & 178,70 \\
\hline 6 & 281,47 & 179,90 & 173,17 & 323,08 & 288,58 & 146,61 & 102,95 & 86,45 & 332,59 & 558,14 & 87,32 & 93,90 \\
\hline 7 & 7,30 & 39,99 & 65,06 & 100,16 & 9,26 & 75,81 & 472,90 & 504,74 & 1,29 & 388,07 & 35,67 & 217,29 \\
\hline 8 & 407,87 & 413,41 & 204,51 & 169,09 & 138,47 & 136,12 & 155,40 & 278,26 & 425,41 & 161,27 & 281,77 & 101,51 \\
\hline 9 & 218,22 & 32,51 & 105,81 & 122,24 & 59,13 & 133,76 & 140,14 & 151,63 & 266,39 & 382,02 & 72,98 & 270,50 \\
\hline 1 & 1 & 63,6 & 19,64 & 138,37 & 68,81 & 86,56 & 607,82 & 30,30 & 334,02 & 184,39 & 8 & 108,24 \\
\hline 11 & 379,47 & 299,32 & 357,56 & 86,17 & 68,37 & 76,26 & 43,96 & 122,84 & 194,34 & 103,71 & 73,86 & 48,72 \\
\hline 12 & 186,87 & 117,55 & 61,45 & 131,70 & 105,96 & 171,49 & 78,66 & 149,23 & 253,92 & 100,79 & 189,12 & 320,91 \\
\hline 13 & 562,98 & 76,59 & 90,03 & 161,20 & 207,66 & 116,03 & 94,82 & 236,85 & 41,72 & 13 & ,36 & 204,60 \\
\hline 14 & 297,14 & 14,78 & 235,98 & 351,86 & 14,59 & 173,69 & 109,53 & 69,14 & 61,41 & 164,82 & 167,59 & 359,26 \\
\hline 15 & 037,12 & 302,67 & 168,94 & 83,38 & 164,55 & 85,70 & 91,62 & 95,10 & 102,53 & 185,67 & 113,80 & 163,40 \\
\hline 16 & 788,26 & 99,12 & 348,13 & 171,70 & 26,46 & 109,07 & 164 & 6 & 17 & 85 & 5 & 37 \\
\hline 17 & 232,90 & 924 & 19,55 & 139,10 & 279,91 & 247,32 & 142 & 205,12 & 89,58 & 86 & 8 & 20 \\
\hline 18 & 548,34 & 432,85 & 167,40 & 352,79 & 48,10 & 326,42 & 211,69 & 253,87 & 681,04 & 319,97 & 260,32 & 207,05 \\
\hline 19 & 184,33 & 10,91 & 165,50 & 171,12 & 328,78 & 262,48 & 184,63 & 114,89 & 297,86 & 57 & 99 & 85,05 \\
\hline 2 & 530,01 & 54,74 & 51,86 & 155,21 & 15,52 &, 19 & 97 & 9 & 7 & 41 & 0 & 7,49 \\
\hline \multicolumn{13}{|l|}{$A L$} \\
\hline 1 & 1117 & 77,07 & 110,10 & 609,17 & 896,98 & 261,64 & 212 & 156,14 & 437,91 & 78,56 & 42,56 & 41,59 \\
\hline 2 & & (19, & 00,00 & (1), & הוע, & $2+0,00$ & 2 & 90,78 & 0 &, 40 & 395,11 & (1) \\
\hline 3 & 249,61 & 410,92 & 417,89 & 760,55 & 994,61 & 583,98 & 303,79 & 516,41 & 567,65 & 41 & 6 & 22 \\
\hline 4 & 186,11 & 345,12 & 337,80 & 140,19 & 118,81 & 455,13 & 100,67 & 144,44 & 143,06 & 134,33 & 4 & 110,15 \\
\hline (5) & (N) & 14 & 62,18 & 83,98 & 118,22 & 68,80 & 128,22 & 9 & 124,51 & 63,00 & & 19,21 \\
\hline 6 & 8,37 & 2042 & 85,05 & 491,05 & 69,10 & 26 & 557,51 & 20 & 64 & 9 & & 192,21 \\
\hline 7 & 712,45 & 397,77 & 350,52 & 337,78 & 178,66 & 239,84 & 235,94 & 94,66 & 174,94 & 145,28 & 132,87 & 100,44 \\
\hline 0 & + & 559,33 & 172,17 & + & 445,57 & 571,94 & 290,65 & & & & 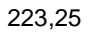 & 25,44 \\
\hline 0 & 7,05 & 19,16 & 31 & 1053,73 & 8 & 47 & 9 & 22 & 72 & 4 & 0 & 17 \\
\hline 10 & 588,35 & 470,97 & 490,53 & 210,51 & 242,95 & 166,08 & 164,53 & 249,44 & 244,20 & 86,53 & 58 & 69,51 \\
\hline 11 & 473,34 & 674,09 & 597,78 & 438,97 & 582,84 & 226,16 & 267,4 & 462,97 & & & & (5<,00 \\
\hline 12 & 026,86 & 368,30 & 15,46 & 104,90 & 08,31 & 368,43 & 86,37 & 76,78 & 185,39 & 187,42 & 8 & $30<>3$ \\
\hline 13 & 372,79 & 616,09 & 171,67 & 590,94 & 605,57 & 711,50 & 295,86 & 574,79 & 345,77 & 132,79 & 323,80 & 173,52 \\
\hline 14 & 597,96 & 711,80 & 691,61 & 812,31 & 947,22 & 760 & 341,21 & & 492,01 & & & 152,08 \\
\hline 15 & 806,00 & 8959 & 577,68 & 694,62 & |51,97 & 150,00 & 990 & 342,23 & 237,21 & 150,55 & ,32 & 138,37 \\
\hline 16 & 59,72 & 88,30 & 79,80 & 98,29 & 164,26 & 61,02 & 61,18 & 58,39 & 54,06 & 85 & 102,86 & 166,97 \\
\hline 17 & 239,67 & 249,30 & 279,40 & 453,78 & 174,08 & 166,49 & 108,25 & 131,09 & 163,50 & 00 & 77 & 92,62 \\
\hline 18 & 2 & 100 & 1 & דו & $2 r$ & 10 & 235,97 & 186,23 & $2<2$ & 3 & 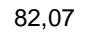 & 210 \\
\hline 19 & 35 & 4 & 39 & 7 & 1 & 6 & 3 & 2 & 9 & 8 & 3 & 15 \\
\hline 20 & 19,74 & 287,90 & 02,48 & 58,58 & 90,31 & 682,46 & 66,31 & 476,28 & 473,81 & 67,49 & 339,90 & 2,4 \\
\hline
\end{tabular}


ANEXO II - continuação.

\begin{tabular}{|c|c|c|c|c|c|c|c|c|c|c|c|c|}
\hline \\
\hline 1 & . & r & 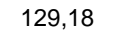 & , & 209,87 & 4,90 & 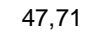 & 10,44 & 0,09 & 59 & ,80 & 100,0 \\
\hline & 3,7 & 6,63 & $c$ & 123,54 & 39 & 44 & 43,87 & 6,85 & 29,75 & 7,05 & 62,66 & $2147 ?$ \\
\hline 3 & 96,66 & 9 & 5 & 141,11 & 3 & 6 & 5 & 1 & 37 & 2 & 305,44 & 99,93 \\
\hline 4 & 912,48 & 247,04 & 392,58 & 292,61 & 675,51 & 881,40 & 252,89 & 394,15 & 303,68 & 649,24 & 110,56 & 449,97 \\
\hline 5 & 531,65 & 124,50 & 114,12 & 71,64 & 320,51 & 135,88 & 79,84 & 229,29 & 8,31 & 258,28 & 147,85 & 272,14 \\
\hline 6 & 455,8 & 82,55 & 100,34 & 54,50 & 345,64 & 65,27 & 163,57 & 329,25 & 953,36 & 95 & 6,59 & 1,99 \\
\hline 7 & & & 590,39 & 222,57 & & & & & 447,97 & 377,71 & 356,07 & 58 \\
\hline 8 & 283,79 & 68,39 & 279,44 & 45,80 & 204,80 & 303,55 & 655,38 & 204,02 & 556,51 & 179,39 & 133,34 & 235,24 \\
\hline 9 & 235,47 & 209,35 & 662,49 & 126,70 & 530,79 & 215,02 & 227,11 & 394,15 & 230,23 & 87,55 & 105,84 & 55,45 \\
\hline 10 & 116,32 & 42,82 & 58,46 & 43,72 & 116,25 & 90,83 & 153,48 & 169,03 & 125,08 & 124,92 & 128,25 & 0,42 \\
\hline 11 & 188,27 & 117,64 & 69,29 & 207,08 & 159,35 & 91,97 & 192,69 & 28,68 & 123,05 & 67 & 127,94 & 7,38 \\
\hline 12 & 657,30 & 1038,87 & 208,86 & 129,61 & 677,44 & 369,46 & 304,99 & 684,82 & 349,72 & 800,08 & 292,09 & 1028,0 \\
\hline 13 & 208,20 & 302,59 & 85,18 & 80,25 & 580,27 & 30,29 & 151,91 & 708,07 & 385,89 & 147,41 & 251,11 & 70,07 \\
\hline 14 & 70,32 & 169,05 & 100,06 & 127,48 & 293,68 & 328,24 & 368,77 & 283,24 & 396,48 & 77,25 & 190,92 & 90,51 \\
\hline 15 & 125,11 & 55,19 & 90,28 & 136,74 & 265,87 & 116,58 & 256,48 & 90,39 & 84,72 & 43,96 & 104,75 & 184,81 \\
\hline 16 & 180,10 & 252,68 & 139,50 & 286,71 & 42,97 & 374,91 & 172,38 & 525,82 & 100,65 & 147,86 & 5,00 & 57,92 \\
\hline 1 & 607,37 & 261,24 & 165,66 & 145,35 & 350,57 & 157,44 & 119,23 & 175,66 & 194,46 & 221,40 & 161,56 & 8,46 \\
\hline 18 & 134,38 & 103,58 & 66,09 & 104,66 & 158,00 & 207,05 & 122,53 & 35,50 & 151,21 & 4,87 & 5,61 & 119,06 \\
\hline 19 & 509,78 & 230,27 & 350,18 & 171,83 & 619,44 & 883,23 & 721,21 & 597,44 & 157,74 & 181,50 & 108,46 & 156,42 \\
\hline 2 & 2,16 & 145,76 &, 99 & 94,83 & 702,93 & 42,51 & 401,18 & 813,15 & 337,05 & 64,84 & 100,79 & 49,91 \\
\hline \multicolumn{13}{|l|}{0} \\
\hline 1 & 487,90 & 489,57 & 34,23 & 45,73 & 597,84 & 195,50 & 162,65 & 198,34 & 368,91 & 348,29 & 259,01 & 162,38 \\
\hline 2 & 340,83 & 408,50 & 683,12 & 693,57 & 878,55 & 232,29 & 107,68 & 94,37 & 233,96 & 1000,52 & 92,26 & 933,12 \\
\hline 3 & 318,75 & 120,21 & 1043,57 & 187,03 & 196,58 & 112,50 & 193,29 & 280,99 & 310,18 & 228,80 & 10,54 & 3,47 \\
\hline 4 & 563,62 & 444,88 & 433,79 & 101,26 & 114,68 & 73,66 &, 13 & 66,66 & 189,46 & 100,71 & & 2,4 \\
\hline 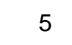 & 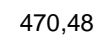 & 450,34 & & & & & 95,61 & & & & & \\
\hline 6 & 7,7 & 51,59 & 3,89 & 6,37 & 41,2 & 117,34 & 65,36 & 66,68 & 65,68 & & & 15 \\
\hline 7 & 798,10 & 400,10 & 686,71 & 800,81 & 348,27 & 98,77 & 100,76 & 107,58 & 169,98 & 102,70 & 4,99 & 09,39 \\
\hline 8 & 7.41 & 913,68 & 486,90 & 454,51 & 230,17 & 104,50 & 85,81 & 153,20 & 2 & 0,60 & 1 & 142,84 \\
\hline 9 & 0,0 & 632,2 & 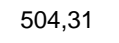 & 0 & 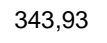 & 0,2 & 39,61 & ,i & & & & 93 \\
\hline 10 & 826,56 & 8 & 546,88 & 720 & 107,56 & 42,64 & 69,31 & 66,88 & 559,02 & & & \\
\hline 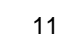 & 576,32 & 201, & 770 & 65 & 338 & 143,11 & $17 €$ & 15 & 72 & 23 & 37 & Do \\
\hline 12 & 46 & 341,16 & 36521 & 102,63 & 241,19 & 242,42 & 130,27 & 171,04 & 63 & 327,90 & 5 & 146,55 \\
\hline 13 & 7,9 & 437,1 & 3,1 & 288,74 & 112,73 & 246,97 & 5,25 & 226,00 & 145,57 & 3 & 59 & 39,35 \\
\hline 14 & 1,0 & 756,49 & - & 766,20 & 571,69 & 34 & 22,09 & 19 & 32 & 06 &, 50 & 84 \\
\hline 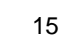 & 11,99 & 977,42 & 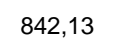 & 698,80 & 643,84 & 217,42 & 6 & 88 & 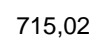 & 15,46 &, 48 & 570,78 \\
\hline 16 & & 65 & 266,79 & 58,92 & 84,15 & 7 & & 40 & 0 & 1 & 2 & 81,41 \\
\hline 11 & $5, / 8$ & 1067,59 & 1079,57 & 720,00 & 107,05 & 888,15 & 731,59 & 723,84 & 749,42 & 574,76 & 527,79 & 466,81 \\
\hline 18 & 159,92 & 315,25 & 295,89 & & & & 138,19 & 141,14 & 387,88 & 291,11 & 2,63 & 365,90 \\
\hline 19 & 1,35 & 90,8 & 175,16 & 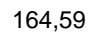 & $4 \mathrm{~s}$ & 0,50 & 0217 & 64,86 & 127,30 & 564,32 & 117,38 & 22,15 \\
\hline 20 & 649,70 & 326,56 & 175,63 & 2,07 & 56,85 & & & & & & 00 & 66,80 \\
\hline
\end{tabular}


ANEXO II - continuação.

\section{Erro Constante}

\begin{tabular}{|c|c|c|c|c|c|c|c|c|c|c|c|c|}
\hline Sujeito & E1 & E2 & E3 & E4 & E5 & E6 & E7 & E8 & $\mathrm{A} 1$ & A2 & A3 & A4 \\
\hline \multicolumn{13}{|l|}{$\mathrm{CO}$} \\
\hline 1 & 24,00 & 9,38 & 162,14 & 66,00 & 109,50 & 21,63 & 18,86 & 118,78 & $-55,00$ & 44,00 & 217,33 & 111,50 \\
\hline 2 & 468,33 & 587,67 & 278,00 & 280,50 & 195,14 & 171,00 & 287,57 & 60,50 & 594,00 & 267,60 & 325,80 & $-5,71$ \\
\hline 3 & 294,50 & 135,00 & 199,60 & 260,00 & 21,43 & 78,40 & 38,75 & 609,86 & 309,33 & 77,50 & 154,25 & 161,33 \\
\hline 4 & 560,50 & 283,00 & 329,00 & 352,00 & 161,00 & 70,40 & 346,33 & 500,80 & 694,67 & 82,50 & 4,20 & 216,29 \\
\hline 5 & 193,14 & 147,00 & 133,80 & 53,00 & 66,50 & $-32,86$ & $-61,50$ & $-14,11$ & 15,33 & 136,00 & $-10,00$ & 74,43 \\
\hline 6 & 486,40 & 589,25 & 320,86 & 165,86 & 190,43 & $-46,71$ & $-42,17$ & $-4,33$ & 378,67 & 316,71 & 88,75 & 3,33 \\
\hline 7 & 342,20 & 396,57 & 312,00 & 89,38 & 87,63 & 72,25 & 429,78 & 322,13 & 820,60 & 717,13 & 326,25 & 31,89 \\
\hline 8 & 1253,57 & 1314,67 & 1082,50 & 736,78 & 481,56 & 501,25 & 293,50 & 293,44 & 1285,75 & 908,56 & 784,56 & 768,33 \\
\hline 9 & $-72,00$ & 31,75 & 86,88 & 106,67 & 13,25 & 76,25 & 123,13 & 171,17 & 329,71 & 211,57 & 229,80 & 210,43 \\
\hline 10 & 139,40 & $-30,00$ & $-29,13$ & $-6,33$ & 12,25 & $-19,13$ & 179,67 & $-31,00$ & 742,75 & 302,00 & $\begin{array}{c}206,78 \\
-\end{array}$ & 98,33 \\
\hline 11 & 58,00 & 45,33 & 67,60 & $-37,00$ & $-97,13$ & $-41,25$ & $\begin{array}{c}-17,63 \\
-\end{array}$ & 4,38 & 41,00 & $-96,71$ & $\begin{array}{c}134,13 \\
-\end{array}$ & 39,00 \\
\hline 12 & $-77,75$ & $-70,25$ & $-10,75$ & $-17,57$ & $-52,57$ & 37,11 & 101,86 & $-76,78$ & 283,83 & 21,00 & 126,43 & 05,75 \\
\hline 13 & 684,83 & 62,11 & $-73,22$ & 54,88 & 95,50 & 17,56 & 68,89 & 107,71 & 208,50 & 14,50 & 109,78 & 145,75 \\
\hline 14 & 358,83 & 620,86 & 64,50 & 23,57 & $-73,29$ & 1,50 & $\begin{array}{c}-10,25 \\
-\end{array}$ & $-53,43$ & 221,00 & 91,13 & $-33,00$ & 266,43 \\
\hline 15 & 379,20 & 81,63 & $-103,67$ & $-4,40$ & $-28,50$ & $-71,50$ & 113,50 & $-88,25$ & 211,50 & $-6,40$ & $-12,17$ & $-9,11$ \\
\hline 16 & 1472,00 & 360,25 & 134,89 & 37,75 & 0,17 & $-21,22$ & 31,71 & 60,75 & 1148,50 & 1498,00 & 174,88 & 60,57 \\
\hline 17 & 421,67 & 455,80 & 513,00 & 373,44 & 295,83 & 362,88 & 396,33 & 458,86 & 431,17 & 627,71 & 446,25 & 411,14 \\
\hline 18 & 713,29 & 245,00 & $-2,25$ & $-16,11$ & $-61,56$ & 102,89 & $-32,88$ & 32,86 & 768,60 & 252,67 & 56,00 & 134,00 \\
\hline 19 & 235,57 & 104,44 & 89,71 & 165,00 & 215,00 & 130,22 & 37,00 & 46,67 & 391,38 & 149,75 & 152,89 & $-6,33$ \\
\hline 20 & 7,00 & 32,14 & $-14,13$ & 68,43 & $-40,13$ & 45,22 & 56,89 & 13,00 & 406,33 & 248,50 & $-25,89$ & 98,00 \\
\hline \multicolumn{13}{|l|}{$A L$} \\
\hline 1 & 1311,50 & 157,50 & 13,25 & 530,00 & 573,63 & 115,33 & 51,57 & 3,29 & 177,14 & $-24,67$ & $-8,33$ & $-22,22$ \\
\hline 2 & 300 & 30425 & 17033 & 21475 & 89,75 & 20033 & 80,00 & 21,33 & -633 & $-71,50$ & 126,00 & $\begin{array}{c}-49,50 \\
-\end{array}$ \\
\hline 3 & 2493,50 & 1829,86 & 1707,60 & 1174,25 & 638,00 & 243,75 & $-10,75$ & $-56,67$ & 149,17 & 49,50 & 128,75 & 194,33 \\
\hline 4 & 234,00 & 258,43 & 404,40 & 164,14 & 49,75 & 111,13 & $-8,71$ & 53,57 & 47,67 & $-21,75$ & 0,86 & $-17,67$ \\
\hline 5 & $-44,80$ & 93,43 & 5,40 & $-21,80$ & $-29,43$ & $-16,50$ & $-25,89$ & 1,50 & $-32,56$ & $-108,14$ & $-69,43$ & $-27,89$ \\
\hline 0 & 140,00 & 498,00 & (1), ט & 879,00 & 000,00 & 735,75 & 531,83 & 348,80 & 143,00 & $-87,14$ & $-44,13$ & 141,56 \\
\hline 7 & 3 & 762,13 & 539,13 & 442,14 & 114,50 & 244,78 & 167,25 & 112 & 158 & 104 & 23 & 66,63 \\
\hline 8 & 1305,00 & 389,86 & 100,40 & 374,60 & 275,80 & 924,43 & 401,33 & 262,88 & 155,60 & 107,86 & $\begin{array}{c}90,67 \\
-\end{array}$ & 321,88 \\
\hline 9 & 1826,00 & 1581,00 & 1071,25 & 1319,17 & 446,11 & 169,78 & 36,63 & 9,22 & 273,00 & $-177,13$ & 110,00 & $-68,25$ \\
\hline 10 & 1321,43 & 836,00 & $115,<0$ & $10<, 11$ & 83,56 & $1<, 00$ & 60,67 & 125,56 & 111,67 & $-172,44$ & $-32,00$ & $-39,00$ \\
\hline 11 & 1211,33 & 1318,33 & 1348,50 & 819,13 & 948,43 & 753,44 & 786,56 & 875,44 & 445,00 & 658,60 & 129,00 & 109,67 \\
\hline 12 & 476,33 & 475,22 & 90,88 & 42,57 & 90,75 & 116,89 & $-40,50$ & $-9,00$ & $-30,50$ & $-24,78$ & $-52,00$ & $-68,22$ \\
\hline 10 & 1681,00 & $1 / 00,01$ & 937,75 & 1295,50 & 000,40 & $3<<, I 3$ & 217,00 & 707,00 & 539,25 & $-118,83$ & 0,10 & $-19,78$ \\
\hline 14 & 865,60 & 1044,67 & 880,50 & 952,78 & 1031,25 & 574,22 & 253,57 & 363,00 & 541,60 & $-16,83$ & $\begin{array}{c}-12,71 \\
-\end{array}$ & $-93,71$ \\
\hline 15 & 492,50 & 659,57 & 541,63 & 433,44 & 193,11 & 163,67 & $-31,00$ & 330,00 & 30,88 & $-88,33$ & 135,22 & $-31,00$ \\
\hline 16 & $-6,75$ & $-19,11$ & 9,33 & 28,44 & 4,38 & $-34,78$ & $-3,50$ & $-30,25$ & $-19,50$ & $-28,89$ & $-46,00$ & 9,22 \\
\hline 17 & 260,67 & 268,00 & 403,50 & 291,57 & 99,89 & 52,43 & 19,25 & 10,78 & 52,57 & $-35,88$ & $-13,89$ & $-53,00$ \\
\hline 18 & 563,83 & 897,67 & 929,50 & 799,63 & 153,83 & 131,56 & 201,75 & 90,43 & 107,38 & 60,25 & $-83,00$ & 70,50 \\
\hline 19 & 1004,17 & 992,40 & 61,17 & 444,67 & 544,83 & 238,25 & 43,71 & 167,29 & 586,29 & $-69,88$ & 54,83 & $-37,71$ \\
\hline 20 & 1610,29 & 1332,00 & 1213,63 & 1149,57 & 1107,63 & 481,75 & 736,29 & 584,33 & 1104,63 & 480,22 & 319,33 & 239,67 \\
\hline
\end{tabular}


ANEXO II - continuação.

\begin{tabular}{|c|c|c|c|c|c|c|c|c|c|c|c|c|}
\hline \multicolumn{13}{|l|}{ I-A } \\
\hline 1 &, 75 & ,20 & משר & 33 & 2,00 & 年 & , & 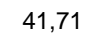 & 0,00 & $-5,00$ & 7,14 & 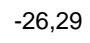 \\
\hline 2 & 23,13 & 4.43 & 2,00 & 2,22 & 91,71 & 1832,86 & 573,67 & 1852,63 & 33,89 & 76,44 & 07,67 & 63,13 \\
\hline 3 & 49,83 & 40,80 & 9,75 & 0,00 & 84,14 & 897,40 & 0,38 & 986,00 & 760,50 & 76,88 & 292,00 & 5,57 \\
\hline 2 & 1039,00 & 340,50 & 8,67 & 337,67 & 416,00 & 1040,00 & 37,60 & 696,50 & 52,71 & 71,00 & 545,25 & 550,00 \\
\hline 5 & 597,00 & 70,86 & 25,17 & 0,57 & 143,33 & 19,50 & $-11,00$ & 167,67 & 13,78 & 25,14 & 58,71 & 28,14 \\
\hline 6 & 36,50 & $-68,6$ & 28,75 & $-57,33$ & 17 , & 26 , & 108 & 163,71 & 333,50 & $-66,80$ & 71,67 & $-57,00$ \\
\hline 7 & & & 631,57 & 218,63 & & & & & 1065,00 & 386,56 & 348,67 & 216,38 \\
\hline 8 & 151,56 & $-20,89$ & 88,57 & 19,11 & 98,33 & 221,00 & 565,00 & 263,00 & 375,88 & 97,11 & 54,22 & 91,38 \\
\hline 9 & 27,50 & 119,89 & 21 , & $-9,22$ & 495 & 120,75 & 181 & 178,67 & 156,86 & -59 & $-49,67$ & 101,33 \\
\hline 10 & 49,88 & $-29,33$ & $-16,89$ & $-48,78$ & 3,17 & 79,00 & $-1,38$ & 107,86 & $-10,17$ & $-7,75$ & $-78,86$ & $-32,11$ \\
\hline . & 81,60 & 87,00 & 8,25 & 58,78 & 104,43 & $-33,43$ & 82,33 & $-49,60$ & $-26,25$ & $-3,43$ & $-64,86$ & $-98,00$ \\
\hline 12 & 1461,33 & 1051,60 & 40,00 & 166,67 & 946,50 & 1778,40 & 2569,40 & 1636,33 & 1044,40 & 976,33 & 358,83 & 498,40 \\
\hline 13 & 176,00 & 57,86 & $-37,25$ & $-44,89$ & 279.67 & 16,86 & 8.75 & 7 & 0 & $\begin{array}{c}68,38 \\
-\end{array}$ & 5 & 00 \\
\hline 1 & 108, & $-107,00$ & -76 & 8,20 & 202,17 & 64 & 199 & 104 & 0 & & 96,00 & \\
\hline 15 & 09,6 & 94,50 & 10,78 & 29,11 & 94,00 & $-253,33$ & $-64,20$ & $-151,17$ & 115 & 126,22 & $-27,86$ & $-45,29$ \\
\hline 16 & 18,80 & $-6,50$ & 50,00 & 14,00 & 0 & 127 & -92 & 211,71 & 40 & 67,89 & 14,25 & $-47,67$ \\
\hline 17 & 647,60 & 175,67 & 141,89 & 44,67 & 439,56 & 281,86 & 90,0 & 187,50 & 245,67 & 62,67 & 44,00 & 1,63 \\
\hline 18 & 43,00 & 27,80 & $-18,00$ & 14,38 & 116,83 & 113,63 & 50,67 & 15,25 & 59 & 38,78 & 4,57 & 21,00 \\
\hline 19 & 0,00 & 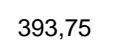 & Th, & 10,00 & 50,50 & 1100,00 & 000 & 400,00 & 230,17 & 39,88 & & \\
\hline & 503,78 & & $-72,50$ & 5723 & & & 23050 & & & & 3 & 196,50 \\
\hline \multicolumn{13}{|l|}{ ( } \\
\hline 1 & 3,00 & 958,43 & 1053,50 & 05,00 & 537,83 & 235,00 & 147 & 99,63 & 317 & 300,43 & 203,13 & 178,71 \\
\hline 2 & 2076,80 & 171,25 & 1225,50 & 057,00 & 583,11 & 109,50 & 45,43 & $-15,50$ & 295,40 & 401,25 & 8,60 & 550,13 \\
\hline 3 & 35,67 & 04,00 & 643,57 & 2,80 & 239,00 & 80 & -44 & $-14,00$ & $-18,00$ & 40 & 7,00 & 76,00 \\
\hline 4 & 2299,50 & 80 & 986 & $1 \varepsilon$ & $-4-4$ & -3 & 16,83 & & & & & 70,50 \\
\hline 5 & 6,7 & 414,25 & 77,75 & 49,00 & 178 & 505 & 448 & 32 & & & 118,56 & 18 \\
\hline 6 & 8,00 & $-33,00$ & $-41,20$ & $-62,17$ & $-38,71$ & $-117,00$ & $-56,89$ & $-47,17$ & $-32,00$ & $-67,67$ & $-52,22$ & 16,60 \\
\hline 7 & 93,25 & 391,80 & 1467,83 & 446,80 & 313,00 & $-99,22$ & $-102,88$ & $-65,40$ & 119,00 & $-64,13$ & $-18,29$ & $-48,00$ \\
\hline 8 & $0<0,11$ & 546,75 & 318,14 & 196,63 & $-25,71$ & & & & & & 5 & \\
\hline 9 & & 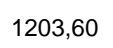 & r & 8 & . & & & & & & & \\
\hline 10 & 1142,38 & 34,89 & 46,78 & 54,22 & (2) & 10 & -8, & 32 , & 7 & -4 & $-23,11$ & 67 \\
\hline 1 & 7,25 & 130,75 & 295,75 & S, & 314 & 47,00 & -20 & 52,3 & 297,33 & 79,67 & 3,63 & $-16,57$ \\
\hline 12 & 838,33 & 1612,14 & 1191,20 & ז & 100, & 300,70 & 608 & $0<4,44$ & 1051,50 & 807,38 & 642,11 & 616,67 \\
\hline & 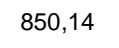 & 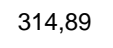 & 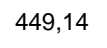 & 81,13 & $-90,89$ & 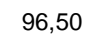 & $-109,22$ & 70 & 00,00 & & 98,60 & 50 \\
\hline 14 & 52,75 & 20,00 & 1282,67 & 3 & 6 & 275,44 & 60,86 & 135,13 & 9 & 4 & 7 & 67,25 \\
\hline 15 & $-593,22$ & 93,44 & $-266,89$ & 50,22 & $-48 /, 44$ & 111 & -43 & -29 & -8 & 44 & 38,00 & 44 \\
\hline 16 & 76,00 & 1258,20 & 17,00 & 727,00 & 7,50 & $482, \mathrm{C}$ & 7,00 & 251,86 & 712,25 & 465,00 & 223,80 & 415,83 \\
\hline 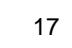 & 年 & Ju,LL & (2) & 0 & 年 & - & ו ו & & 104,00 & 20,70 & o, & . \\
\hline 10 & 7 & 782,40 & 238,20 & 159,00 & & & & & & & 6,00 & \\
\hline & & & & -81 & & & & & & & 101,00 & $-35,33$ \\
\hline ว & 607,86 & 244,60 & 30,63 & 33,86 & $-55,00$ & $-66,86$ & $-44,43$ & $-40,33$ & $-63,71$ & $-44,25$ & $-36,00$ & $-66,78$ \\
\hline
\end{tabular}


ANEXO III - Termo de consentimento livre e esclarecido.

ESCOLA DE EDUCAÇÃO FÍSICA E ESPORTE DA UNIVERSIDADE DE SÃO PAULO Comitê de Ética em Pesquisa

TERMO DE CONSENTIMENTO LIVRE E ESCLARECIDO (Instruções para preenchimento ao final)

I - DADOS DE IDENTIFICAÇÃO DO SUJEITO DA PESQUISA OU RESPONSÁVEL LEGAL

1. NOME DO INDIVÍDUO:

Documento de identidade №.

Sexo: $\square \mathrm{M} \quad \square \mathrm{F}$

Data de nascimento:

Endereço:

№

APTO

Bairro:

Cidade:

CEP:

Telefone:

\section{RESPONSÁVEL LEGAL:}

Natureza (grau de parentesco, tutor, curador, etc.)

Documento de identidade №:

Sexo: $\square \mathrm{M} \quad \square \mathrm{F}$

Data de nascimento:

Endereço:

№

APTO

Bairro:

Cidade:

CEP:

Telefone:

II - DADOS SOBRE A PESQUISA CIENTÍFICA

1. Título do Projeto de Pesquisa

Estrutura da prática e complexidade da tarefa em aprendizagem motora

2. Pesquisador Responsável

PROF. DR. Umberto César Corrêa 
3. Cargo/Função

Livre docente

4. Avaliação do risco da pesquisa:

RISCO MÍNIMO $\bigotimes \quad$ RISCO BAIXO $\square \quad$ RISCO MÉDIO $\square \quad$ RISCO MAIOR

(probabilidade de que o indivíduo sofra algum dano como conseqüência imediata ou tardia do estudo)

5. Duração da Pesquisa

Março a Setembro de 2008

III - EXPLICAÇÕES DO PESQUISADOR AO INDIVÍDUO OU SEU REPRESENTANTE LEGAL SOBRE A PESQUISA, DE FORMA CLARA E SIMPLES, CONSIGNANDO:

1. Esse estudo está sendo desenvolvido pelo Laboratório de Comportamento Motor da Escola de Educação Física e Esporte da USP com o objetivo de investigar os efeitos da estrutura de prática e da complexidade da tarefa na aprendizagem de habilidades motoras.

2. Você vai praticar uma tarefa, na qual será realizada uma seqüência de toques em sensores dispostos sobre uma mesa de madeira, para que um estudo sobre qual o efeito da estrutura de prática em tarefa com diferentes níveis de complexidade, seja realizado.

3. Não há desconfortos e riscos esperados;

4. Você poderá manter parte do desempenho obtido na tarefa do estudo em tarefas semelhantes no dia a dia.

IV - ESCLARECIMENTOS DADOS PELO PESQUISADOR SOBRE GARANTIAS DO SUJEITO DA PESQUISA:

1. A criança e seu responsável podem, em qualquer momento, ter a informação que desejarem a respeito de procedimentos, eventuais riscos e benefícios relacionados à pesquisa;

2. A criança e seu responsável têm a liberdade de retirar seu consentimento e de deixar de participar do estudo, sem nenhum prejuízo;

3. Nenhuma informação a respeito da identidade da criança ou do responsável serão tornadas públicas;

4. Será providenciada assistência por eventuais danos à saúde decorrentes da pesquisa. 
$V$ - INFORMAÇÕES DE NOMES, ENDEREÇOS E TELEFONES DOS RESPONSÁVEIS PELO ACOMPANHAMENTO DA PESQUISA, PARA CONTATO EM CASO DE INTERCORRÊNCIAS CLÍNICAS E REAÇÕES ADVERSAS.

Para questões associadas com este experimento, por favor, entre em contato com Prof. Dr. Umberto César Corrêa -, pesquisador responsável pelo estudo pelo telefone (11) 3091-2147. O Laboratório de Comportamento Motor fica na escola de Educação Física e Esporte, Universidade de São Paulo (USP), na avenida Prof. Dr. Mello Moraes, 65 - Butantã, São Paulo, SP. CEP 05508-030, fone (11) 3091-2147.

\section{VI. - OBSERVAÇÕES COMPLEMENTARES} Não há.

VII - CONSENTIMENTO PÓS-ESCLARECIDO

Declaro que, após convenientemente esclarecido pelo pesquisador e ter entendido o que me foi explicado, consinto em participar do presente Projeto de Pesquisa.

Pouso Alegre, de de 20

assinatura do sujeito da pesquisa

ou responsável legal assinatura do pesquisador

(carimbo ou nome legível) 